\title{
REPRESENTATION STABILITY FOR FILTRATIONS OF TORELLI GROUPS
}

\author{
PETER PATZT
}

\begin{abstract}
We show, finitely generated rational $\mathrm{VIC}_{\mathbb{Q}}-$ modules and $\mathrm{SI}_{\mathbb{Q}}-$ modules are uniformly representation stable and all their submodules are finitely generated. We use this to prove two conjectures of Church and Farb, which state that the quotients of the lower central series of the Torelli subgroups of $\operatorname{Aut}\left(F_{n}\right)$ and $\operatorname{Mod}\left(\Sigma_{g, 1}\right)$ are uniformly representation stable as sequences of representations of the general linear groups and the symplectic groups, respectively. Furthermore we prove an analogous statement for their Johnson filtrations.
\end{abstract}

\section{INTRODUCTION}

Church and Farb [CF13] define the notion of representation stability for sequences of representations of the symmetric groups $\mathfrak{S}_{n}$, the hyperoctahedral groups $\mathbb{Z} \rtimes \mathfrak{S}_{n}$, the general linear groups $\mathrm{GL}_{n} \mathbb{Q}$, the special linear groups $\mathrm{SL}_{n} \mathbb{Q}$ and the symplectic groups $\mathrm{Sp}_{2 n} \mathbb{Q}$. Especially representation stability for the symmetric groups has been the focus of a lot of research lately. It has been intimately connected to functors from the category of finite sets and injections FI to vector spaces by Church, Ellenberg and Farb [CEF15]. Wilson [Wil14] developed a similar connection for the hyperoctahedral groups. In both cases an amplitude of sequences were proved to be representation stable.

Representation stability over the symmetric groups. The representation theory of the symmetric group $\mathfrak{S}_{n}$ over the rationals $\mathbb{Q}$ is known to be semisimple. The irreducible representations are indexed by partitions $\lambda=\left(\lambda_{1} \geq \lambda_{2} \geq \ldots\right)$ of $n=\lambda_{1}+\lambda_{2}+\ldots$, which we denote by

$$
\mathfrak{S}_{n}(\lambda)
$$

Let

$$
V_{0} \stackrel{\phi_{0}}{\longrightarrow} V_{1} \stackrel{\phi_{1}}{\longrightarrow} V_{2} \stackrel{\phi_{2}}{\longrightarrow} \ldots
$$

be a sequence of vector spaces over $\mathbb{Q}$ together with a linear $\mathfrak{S}_{n}$-action on $V_{n}$ such that $\phi_{n}$ is $\mathfrak{S}_{n}$-equivariant. Such a sequence is called consistent by Church-Farb [CF13] and can easily be generalized to the other groups mentioned in the first paragraph. They [CF13, Def 2.3] call a consistent sequence of representations of the symmetric groups representation stable if the following conditions are satisfied:

Date: April 2017.

2010 Mathematics Subject Classification. 20G05 (Primary), 20E36, 20F40, 58D05 (Secondary). 
Injectivity: The map $\phi_{n}: V_{n} \rightarrow V_{n+1}$ is injective for all large enough $n \in \mathbb{N}$.

Surjectivity: The induced map $\operatorname{Ind}_{\mathfrak{S}_{n}}^{\mathfrak{S}_{n+1}} \phi_{n}: \operatorname{Ind}_{\mathfrak{S}_{n}}^{\mathfrak{S}_{n+1}} V_{n} \rightarrow V_{n+1}$ is surjective for all large enough $n \in \mathbb{N}$.

Multiplicity stability: We can write

$$
V_{n} \cong \bigoplus_{\lambda} \mathfrak{S}_{n}(\lambda)^{\oplus c_{\tilde{\lambda}, n}}
$$

where $\tilde{\lambda}=\left(\lambda_{2} \geq \lambda_{3} \geq \ldots\right)$ for $\lambda=\left(\lambda_{1} \geq \lambda_{2} \geq \lambda_{3} \geq \ldots\right)$ and $c_{\tilde{\lambda}, n}$ is independent of $n$ for all large enough $n \in \mathbb{N}$.

A consistent sequence is called uniformly representation stable if the multiplicities $c_{\tilde{\lambda}, n}$ stabilize uniformly.

Functors from a category $\mathcal{C}$ to the category $\mathbb{Q}$-mod of vector spaces over $\mathbb{Q}$ are called $\mathcal{C}$-modules. Every $\mathrm{FI}$-module $V: \mathrm{FI} \rightarrow \mathbb{Q}$-mod gives rise to a consistent sequence, by taking

$$
V_{n}=V(\{1, \ldots, n\})
$$

and

$$
\phi_{n}=V(\{1, \ldots, n\} \rightarrow\{1, \ldots, n+1\}) .
$$

The connection to representation stability was provided by Church-Ellenberg-Farb in the following theorem.

Theorem (Church-Ellenberg-Farb [CEF15, Thm 1.13]). An FI-module $V$ is finitely generated if and only if its consistent sequence is uniformly representation stable and $V_{n}$ is finite dimensional for all $n \in \mathbb{N}$.

This theorem depends on the following noetherian property of $\mathrm{FI}$-modules.

Theorem (Church-Ellenberg-Farb [CEF15, Thm 1.3]). Every submodule of a finitely generated $\mathrm{FI}-$ module is finitely generated.

Analogous theorems for the hyperoctahedral groups were proved by Wilson [Wil14, Thm $4.21+$ Thm 4.22].

Representation stability over the general linear groups and symplectic groups. The rational representation theory for both $\mathrm{GL}_{n} \mathbb{Q}$ and $\mathrm{Sp}_{2 n} \mathbb{Q}$ is semisimple and the irreducibles are indexed by pairs of partitions $\left(\lambda^{+}, \lambda^{-}\right)$such that the lengths $\ell\left(\lambda^{+}\right)+\ell\left(\lambda^{-}\right) \leq n$ and by partitions $\lambda$ whose length $\ell(\lambda) \leq n$, respectively. We respectively denote these irreducibles by

$$
\mathrm{GL}_{n}\left(\lambda^{+}, \lambda^{-}\right) \text {and } \operatorname{Sp}_{2 n}(\lambda) \text {. }
$$

For a consistent sequence of rational representations of the general linear groups or the symplectic groups Church-Farb [CF13, Def 2.3] define (uniform) representation stability analogously to the symmetric groups - only the analogue of the multiplicity stability condition is easier to state: 
Multiplicity stability for general linear groups: We can write

$$
V_{n} \cong \bigoplus_{\lambda^{+}, \lambda^{-}} \operatorname{GL}_{n}\left(\lambda^{+}, \lambda^{-}\right)^{\oplus c_{\left(\lambda^{+}, \lambda^{-}\right), n}}
$$

and $c_{\left(\lambda^{+}, \lambda^{-}\right), n}$ is independent of $n$ for all large enough $n \in \mathbb{N}$.

Multiplicity stability for symplectic groups: We can write

$$
V_{n} \cong \bigoplus_{\lambda} \operatorname{Sp}_{2 n}(\lambda)^{\oplus c_{\lambda, n}}
$$

and $c_{\lambda, n}$ is independent of $n$ for all large enough $n \in \mathbb{N}$.

The question of the analogue of FI for the general linear groups could be naively answered with $\mathrm{VI}$ - the category of finite dimensional vector spaces and injective homomorphisms. But this is not correct, it turns out that the correct analogue is VIC - the category of finite dimensional vector spaces and injective homomorphisms together with a choice of a complement of the image (see Definition 3.1). For the symplectic groups we use SI- the category of finite dimensional symplectic vector spaces and isometries. This works well, because isometries are always injective and come with a canonical complement. A version of VIC and SI for finite rings has already been used by Putman-Sam [PS14].

Every VIC-module $V:$ VIC $\rightarrow \mathbb{Q}$-mod gives rise to a consistent sequence, by taking

$$
V_{n}=V\left(\mathbb{Q}^{n}\right)
$$

and

$$
\phi_{n}=V\left(\mathbb{Q}^{n} \rightarrow \mathbb{Q}^{n+1}\right) .
$$

Similarly for every SI-module $V: \mathrm{SI} \rightarrow \mathbb{Q}$-mod the sequence given by

$$
V_{n}=V\left(\mathbb{Q}^{2 n}\right)
$$

and

$$
\phi_{n}=V\left(\mathbb{Q}^{2 n} \rightarrow \mathbb{Q}^{2 n+2}\right)
$$

is consistent. We call $V$ rational if $V_{n}$ is a rational representation for every $n \in \mathbb{N}$.

Our main technical results are the following theorems.

Theorem A. A rational VIC-module $V$ is finitely generated if and only if its consistent sequence is uniformly representation stable and $V_{n}$ is finite dimensional for all $n \in \mathbb{N}$.

Theorem B. A rational SI-module $V$ is finitely generated if and only if its consistent sequence is uniformly representation stable and $V_{n}$ is finite dimensional for all $n \in \mathbb{N}$.

We also prove the following noetherian condition.

Theorem C. Every submodule of a finitely generated rational VIC-module is finitely generated. 
Theorem D. Every submodule of a finitely generated rational SI-module is finitely generated.

Remark. (a) We may substitute any field of characteristic zero for $\mathbb{Q}$ and the theorems remain true.

(b) Putman-Sam [PS14] proved analogues of Theorems $C$ and $D$ for finite rings.

(c) Gan-Watterlond [GW16] proved an analogue of Theorem A for finite fields.

Torelli groups. Let $F_{n}$ denote the free group on $n$ generators, then its abelianization is $\mathbb{Z}^{n}$. The quotient map induces an epimorphism on their automorphism groups. The Torelli subgroup $\mathrm{IA}_{n}$ is defined as the kernel, so we get the following short exact sequence.

$$
1 \rightarrow \mathrm{IA}_{n} \rightarrow \operatorname{Aut}\left(F_{n}\right) \rightarrow \operatorname{Aut}\left(\mathbb{Z}^{n}\right) \cong \mathrm{GL}_{n} \mathbb{Z} \rightarrow 1
$$

Let $\Sigma_{g, 1}$ denote the compact, oriented genus $g$ surface with one boundary component. The mapping class group $\operatorname{Mod}\left(\Sigma_{g, 1}\right)$ is the discrete group $\pi_{0} \mathrm{Homeo}^{+}\left(\Sigma_{g, 1}, \partial \Sigma_{g, 1}\right)$ of isotopy classes of orientation-preserving homeomorphisms of $\Sigma_{g, 1}$ that fix the boundary pointwise. The action of $\operatorname{Mod}\left(\Sigma_{g, 1}\right)$ on $H_{1}\left(\Sigma_{g, 1} ; \mathbb{Z}\right) \cong \mathbb{Z}^{2 g}$ is symplectic, and the Torelli subgroup $\mathcal{I}_{g, 1}$ is defined to be the kernel of this action. In fact, there is a short exact sequence

$$
1 \rightarrow \mathcal{I}_{g, 1} \rightarrow \operatorname{Mod}\left(\Sigma_{g, 1}\right) \rightarrow \operatorname{Sp}\left(H_{1}\left(\Sigma_{g, 1} ; \mathbb{Z}\right)\right) \cong \operatorname{Sp}_{2 g}(\mathbb{Z}) \rightarrow 1
$$

Very little is known about the homology of both Torelli subgroups, except in homological degree 1 . The rational homology is conjectured to be uniformly representation stable in [CF13, Conj 6.1, Conj 6.3]. This problem seems to be too hard to tackle right now, as it is not even known whether the rational homology groups are representations of $\mathrm{GL}_{n} \mathbb{Q}$ and $\mathrm{Sp}_{2 g} \mathbb{Q}$.

Another subject of study deals with central series of the Torelli groups, which include the lower central series $\gamma \mathrm{IA}_{n}=\left\{\gamma_{i} \mathrm{IA}_{n}\right\}_{i \in \mathbb{N}}$ and $\gamma \mathcal{I}_{g, 1}$ and the Johnson filtration $\alpha \mathrm{IA}_{n}$ and $\alpha \mathcal{I}_{g, 1}$ (see Section 4 and the beginning of Section 6 ). The information of these central series are compiled nicely in their graded rational Lie algebra $\operatorname{gr}\left(\gamma \mathrm{IA}_{n}\right), \operatorname{gr}\left(\gamma \mathcal{I}_{g, 1}\right), \operatorname{gr}\left(\alpha \mathrm{IA}_{n}\right)$ and $\operatorname{gr}\left(\alpha \mathcal{I}_{g, 1}\right)$ (see Definition 4.2). All of these filtrations were considered before, for example by Andreadakis [And65], Hain [Hai97], Habegger-Sorger [HS00], Satoh [Sat12, Sat16] and are known to be separating, ie

$$
\bigcap_{i \geq 1} \gamma_{i} \mathrm{IA}_{n}=\bigcap_{i \geq 1} \gamma_{i} \mathcal{I}_{g, 1}=\bigcap_{i \geq 1} \alpha_{i} \mathrm{IA}_{n}=\bigcap_{i \geq 1} \alpha_{i} \mathcal{I}_{g, 1}=1 .
$$

Church and Farb [CF13, Conj 6.2 and the paragraph below Conj 6.3] conjectured that each degree of the Lie algebras corresponding to the lower central series is uniformly representation stable. The following theorems address exactly the conjectures as stated by Church and Farb.

Theorem E. For every fixed $i \geq 1$ and $n \in \mathbb{N}$, the natural $\mathrm{GL}_{n} \mathbb{Z}$-representation on the ith quotient of the lower central series $\operatorname{gr}_{i}\left(\gamma \mathrm{IA}_{n}\right)$ extends to a rational $\mathrm{GL}_{n} \mathbb{Q}-$ representation. 
Theorem F. For every fixed $i \geq 1$ the sequence of the ith quotients of the lower central series $\left\{\operatorname{gr}_{i}\left(\gamma \mathrm{IA}_{n}\right)\right\}_{n \in \mathbb{N}}$ of $\mathrm{GL}_{n} \mathbb{Q}$-representations is uniformly representation stable.

Theorem G. For every fixed $i \geq 1$ the sequence of the ith quotients of the lower central series $\left\{\operatorname{gr}_{i}\left(\gamma \mathcal{I}_{g, 1}\right)\right\}_{g \in \mathbb{N}}$ of $\operatorname{Sp}_{2 g} \mathbb{Q}$-representations is uniformly representation stable.

It is noteworthy that if a $\mathrm{GL}_{n} \mathbb{Z}$-representation can be extended to a rational $\mathrm{GL}_{n} \mathbb{Q}$-representation, this extension is not unique. In Section 2.9 it is explained how there are infinitely many different possible extensions. However, a sequence of extensions that satisfies Theorem $\mathrm{F}$ is uniquely determined for all large enough $n \in$ $\mathbb{N}$. To prove Theorem F, we will find the correct way to extend these representations for large enough $n \in \mathbb{N}$. For representations of the symplectic groups this problem does not arise.

We are also able to prove similar results for the Lie algebras corresponding to the Johnson filtrations.

Theorem H. For every fixed $i \geq 1$ the sequence of the ith quotients of the Johnson filtration $\left\{\operatorname{gr}_{i}\left(\alpha \mathrm{IA}_{n}\right)\right\}_{n \in \mathbb{N}}$ of $\mathrm{GL}_{n} \mathbb{Q}$-representations is uniformly representation stable.

Theorem I. For every fixed $i \geq 1$ the sequence of the ith quotients of the Johnson filtration $\left\{\operatorname{gr}_{i}\left(\alpha \mathcal{I}_{g, 1}\right)\right\}_{g \in \mathbb{N}}$ of $\operatorname{Sp}_{2 g} \mathbb{Q}$-representations is uniformly representation stable.

We also prove analogues of Theorem $\mathrm{E}$ for the filtrations $\gamma \mathcal{I}_{g, 1}, \alpha \mathrm{IA}_{n}$ and $\alpha \mathcal{I}_{g, 1}$, although they can already be found in the literature (eg in [HS00, Thm 1.1] and [Sat16]).

This work is the author's PhD thesis.

Acknowledgements. First and foremost the author wishes to thank his advisor Holger Reich for introducing him to the interesting and emerging research on representation stability. During his PhD the author was supported by the Berlin Mathematical School, the SFB Raum-Zeit-Materie and the Dahlem Research School. The author also wants to thank Kevin Casto, Tom Church, Daniela Egas Santander, Benson Farb, Daniel Lütgehetmann, Jeremy Miller, Holger Reich, Steven Sam, David Speyer and Elmar Vogt for helpful conversations. Special thanks to Steven Sam for his extensive help with the modification rules, and to Kevin Casto for pointing out the conjectures to the author.

\section{RAtional REPRESENTAtion THEORY OF THE GENERAL LINEAR GROUPS AND} THE SYMPLECTIC GROUPS

Let us start by shortly recalling the rational (or algebraic) representation theory of the algebraic groups $\mathrm{GL}_{n} \mathbb{Q}$ and $\mathrm{Sp}_{2 n} \mathbb{Q}$. More elaboration can be found in the 
books of Fulton-Harris [FH91], Green [Gre07], Goodman-Wallach [GW09], Jantzen [Jan87], Weyl [Wey39], and the paper of Koike [Koi89].

2.1. Algebraic groups, polynomial and rational representations. In general an algebraic group over a field $k$ is a variety that has a compatible group structure. That means multiplication and inverses are regular maps of varieties. Two simple examples are the additive group $(k,+)$ considered as the affine variety $\mathbb{A}^{1}$ and the multiplicative group $\left(k^{\times}, \cdot\right)$ considered as the subvariety of $\mathbb{A}^{2}$ given by the polynomial $x y=1$.

A more complicated example is the general linear group $\mathrm{GL}_{n}(k)$. To define it, we consider a subvariety of $\mathbb{A}^{n^{2}+1}$. Let its coordinates be denoted by $\left\{x_{i j}\right\}_{i, j=1, \ldots, n}$ and $t$. Then the determinant $\operatorname{det}\left(x_{i j}\right)$ of the matrix given by $\left\{x_{i j}\right\}_{i, j=1, \ldots, n}$ is a polynomial. Let $\mathrm{GL}_{n}(k)$ be the subvariety of $\mathbb{A}^{n^{2}+1}$ given as the zero set of the polynomial $\operatorname{det}\left(x_{i j}\right) \cdot t-1$. The multiplication given by matrix multiplication and the inverse given by Cramer's rule is polynomial. Thus $\mathrm{GL}_{n}(k)$ is an algebraic group.

The symplectic group $\mathrm{Sp}_{2 n}(k)$ is the subgroup of $\mathrm{GL}_{2 n}(k)$ given by those matrices $\left(x_{i j}\right)$ whose inverse is

$$
\left(x_{i j}\right)^{-1}=\Omega_{n} \cdot\left(x_{i j}^{T}\right) \cdot \Omega_{n}^{T}
$$

where $\Omega_{n}$ is the Gram matrix of the standard symplectic form

$$
\Omega_{n}=\left(\begin{array}{ccccc}
0 & 1 & & & \\
-1 & 0 & & & \\
& & \ddots & & \\
& & & 0 & 1 \\
& & & -1 & 0
\end{array}\right)
$$

Therefore the symplectic group is the zero set of the polynomials

$$
\left(x_{i j}\right) \cdot \Omega_{n} \cdot\left(x_{i j}^{T}\right) \cdot \Omega_{n}^{T}-1 .
$$

Thus $\mathrm{Sp}_{2 n}(k)$ is an algebraic group.

A (finite dimensional) polynomial/rational representation of an algebraic group $G$ over $k$ is a map

$$
G \longrightarrow \mathrm{GL}_{n}(k)
$$

for some $n \in \mathbb{N}$ that is a group homomorphism and a polynomial/rational map of varieties. For infinite dimensional representations, one may construct the algebraic group $\mathrm{GL}(V)$ for infinite dimensional vector spaces $V$ over $k$. Note that every polynomial representation is by definition also rational.

Both for $\mathrm{GL}_{n}(k)$ and $\mathrm{Sp}_{2 n}(k)$ there is a standard representation given by

$$
\mathrm{GL}_{n}(k) \stackrel{\mathrm{id}}{\longrightarrow} \mathrm{GL}_{n}(k)
$$

and

$$
\mathrm{Sp}_{2 n}(k) \longleftrightarrow \mathrm{GL}_{2 n}(k)
$$


respectively. Both are polynomial representations.

2.2. The representation theory of $\mathrm{GL}_{n} \mathbb{Q}$. It turns out that both the polynomial representation theory and the rational representation theory of $\mathrm{GL}_{n} \mathbb{Q}$ are semisimple and all irreducible representations are finite dimensional.

The standard representation $V_{n}=\mathbb{Q}^{n}$ is irreducible. All other irreducible polynomial representations can be constructed as subquotients of the $r$-fold tensor product $V_{n}^{\otimes r}$ for some $r \in \mathbb{N}$ on which $\mathrm{GL}_{n} \mathbb{Q}$ acts diagonally. We get a right action of the symmetric group $\mathfrak{S}_{r}$ on $r$ letters on $V_{n}^{\otimes r}$, which makes it a $\mathbb{Q} \mathrm{GL}_{n} \mathbb{Q}-\mathbb{Q S}_{r^{-}}$ bimodule. Let $\lambda$ be a partition of $r$, then $r$ is called the size of $\lambda$ and is denoted by $|\lambda|$. Let $\mathfrak{S}_{r}(\lambda)$ be its associated irreducible Specht module of $\mathbb{Q} \mathfrak{S}_{r}$, then

$$
\mathrm{GL}_{n}(\lambda):=V_{n}^{\otimes r} \underset{\mathbb{Q S}_{r}}{\otimes} \mathfrak{S}_{r}(\lambda)
$$

is an irreducible $\mathrm{GL}_{n} \mathbb{Q}$-representation if $\lambda$ has at most $n$ rows and zero otherwise. We call this number the length of $\lambda$ and denote it by $\ell(\lambda)$. In fact, all irreducible polynomial $\mathrm{GL}_{n} \mathbb{Q}$-representations are isomorphic to $\mathrm{GL}_{n}(\lambda)$ for some partition $\lambda$ with at most $n$ rows and they are up to isomorphism uniquely determined by it.

To get rational representations of $\mathrm{GL}_{n} \mathbb{Q}$, we need to introduce the dual representation $V_{n}^{*}=\operatorname{Hom}_{\mathbb{Q}}\left(\mathbb{Q}^{n}, \mathbb{Q}\right)$ of $V_{n}$, which is defined by $g \cdot f(v)=f\left(g^{-1} \cdot v\right)$. Define furthermore $V_{n}^{\{r, s\}}$ to be the intersection of the kernels of all contraction maps

$$
V_{n}^{\otimes r} \otimes V_{n}^{* \otimes s} \longrightarrow V_{n}^{\otimes r-1} \otimes V_{n}^{* \otimes s-1}
$$

$v_{1} \otimes \cdots \otimes v_{r} \otimes f_{1} \otimes \cdots \otimes f_{s} \longmapsto f_{j}\left(v_{i}\right) \cdot v_{1} \otimes \cdots \otimes \hat{v}_{i} \otimes \cdots \otimes v_{r} \otimes f_{1} \otimes \cdots \otimes \hat{f}_{j} \otimes \cdots \otimes f_{s}$.

Let $\lambda^{+}$be a partition of $r$ and $\lambda^{-}$a partition of $s$. We call $r+s$ the size of the pair $\left(\lambda^{+}, \lambda^{-}\right)$. Then

$$
\operatorname{GL}_{n}\left(\lambda^{+}, \lambda^{-}\right):=V_{n}^{\{r, s\}} \underset{\mathbb{Q S}_{r} \otimes \mathbb{Q S}_{s}}{\otimes}\left(\mathfrak{S}_{r}\left(\lambda^{+}\right) \otimes \mathfrak{S}_{s}\left(\lambda^{-}\right)\right)
$$

is a rational $\mathrm{GL}_{n} \mathbb{Q}$-representation. It is irreducible if the length of the pair $\ell\left(\lambda^{+}\right)+$ $\ell\left(\lambda^{-}\right) \leq n$ and zero otherwise. All irreducible rational $\mathrm{GL}_{n} \mathbb{Q}$-representations are isomorphic to $\mathrm{GL}_{n}\left(\lambda^{+}, \lambda^{-}\right)$for some partitions $\lambda^{+}, \lambda^{-}$which together have at most $n$ rows and they are up to isomorphism uniquely determined by it.

In terms of weights, if $\ell\left(\lambda^{+}\right)+\ell\left(\lambda^{-}\right) \leq n$, the irreducible representation $\mathrm{GL}_{n}\left(\lambda^{+}, \lambda^{-}\right)$ has the highest weight

$$
\left(\lambda_{1}^{+} L_{1}+\lambda_{2}^{+} L_{2}+\ldots\right)-\left(\lambda_{1}^{-} L_{n-1}+\lambda_{2}^{-} L_{n-2}+\ldots\right) .
$$

Here $L_{i} \in \mathfrak{h}^{*}$ are elements of the dual vector space of the $n \times n$ diagonal matrices $\mathfrak{h} \cong \mathbb{Q}^{n}$. The matrices $E_{i, i}$ sending $e_{i}$ to itself and all other $e_{j}$ to zero gives a basis of $\mathfrak{h}$ and

$$
L_{i}\left(E_{j, j}\right)=\delta_{i, j}
$$

gives its dual basis. For more details on the notation see Fulton-Harris [FH91, §15].

Note that $\mathrm{GL}_{n}(\lambda, \emptyset)=\mathrm{GL}_{n}(\lambda)$ is polynomial and $\mathrm{GL}_{n}(\emptyset)$ is the trivial representation. Another significant representation is the one-dimensional determinant 
representation $D$ given by

$$
g \cdot 1=\operatorname{det} g \cdot 1 \text {. }
$$

For each $k \in \mathbb{Z}$ let $D_{k}$ be the one-dimensional representation given by

$$
g \cdot 1=(\operatorname{det} g)^{k} \cdot 1 \text {. }
$$

The highest weight of $D_{k}$ is

$$
k\left(L_{1}+\cdots+L_{n}\right) .
$$

Interestingly, if $V$ is the irreducible $\mathrm{GL}_{n} \mathbb{Q}$-representation with highest weight

$$
\lambda_{1} L_{1}+\cdots+\lambda_{n} L_{n}
$$

for some integers $\lambda_{1} \geq \cdots \geq \lambda_{n}$, then $V \otimes D_{k}$ is irreducible and has the highest weight

$$
\left(\lambda_{1}+k\right) L_{1}+\cdots+\left(\lambda_{n}+k\right) L_{n}
$$

2.3. The representation theory of $\mathrm{Sp}_{n} \mathbb{Q}$. For the symplectic groups every rational representation is already polynomial. As for the general linear groups, the rational representation theory of $\operatorname{Sp}_{2 n} \mathbb{Q}$ is semisimple and every irreducible representation is finite dimensional.

The standard representation $V_{n}=\mathbb{Q}^{2 n}$ is irreducible. All other irreducible rational representations can be constructed as subquotients of the $r$-fold tensor product $V_{n}^{\otimes r}$ for some $r \in \mathbb{N}$ on which $\operatorname{Sp}_{2 n} \mathbb{Q}$ acts diagonally. Let $\langle$,$\rangle denote the symplectic$ form on $V_{n}$. Then for $r \geq 2$ there are contractions

$$
\begin{gathered}
V_{n}^{\otimes r} \longrightarrow V_{n}^{\otimes r-2} \\
v_{1} \otimes \cdots \otimes v_{r} \longmapsto\left\langle v_{i}, v_{j}\right\rangle \cdot v_{1} \otimes \cdots \otimes \hat{v}_{i} \otimes \cdots \otimes \hat{v}_{j} \otimes \cdots \otimes v_{r} .
\end{gathered}
$$

Let $V_{n}^{\langle r\rangle}$ denote the intersection of the kernels of all these maps. Then

$$
\operatorname{Sp}_{2 n}(\lambda):=V_{n}^{\langle r\rangle} \underset{\mathbb{Q} \mathfrak{S}_{r}}{\otimes} \mathfrak{S}_{r}(\lambda)
$$

is a rational $\mathrm{Sp}_{2 n} \mathbb{Q}$-representation. It is irreducible if $\lambda$ has at most $n$ rows and zero otherwise. All irreducible rational $\mathrm{Sp}_{2 n} \mathbb{Q}$-representations are of this form.

In terms of weights, if $\ell(\lambda) \leq n$, the irreducible representation $\operatorname{Sp}_{2 n}(\lambda)$ has the highest weight

$$
\lambda_{1} L_{1}+\lambda_{2} L_{2}+\cdots+\lambda_{n} L_{n} .
$$

Here $L_{i} \in \mathfrak{h}^{*}$ are elements of the dual vector space of the Cartan subalgebra $\mathfrak{h} \cong \mathbb{Q}^{n}$ of the $2 n \times 2 n$ matrices generated by the basis $H_{i}=\left(E_{2 i-1,2 i-1}-E_{2 i, 2 i}\right)$ with $i=1, \ldots, n$. Then $L_{i}$ is the dual basis with

$$
L_{i}\left(H_{j}\right)=\delta_{i, j}
$$

For more details on the notation see [FH91, $\S 16+\S 17]$.

2.4. Littlewood-Richardson coefficients. Subsequently, we will make extensive use of the Littlewood-Richardson coefficients $c_{\mu \nu}^{\lambda}$. These arise in various situations, especially in the context of branching rules, which we wish to cover in 
the next subsections. An introduction to these coefficients can be found in Fulton [Ful97]. For our purpose the following two propositions suffice.

The first proposition showcases the role of the Littlewood-Richardson coefficients in branching rules. Throughout the paper we use the abbreviation

$$
[V, W]=\operatorname{dim} \operatorname{Hom}_{G}(V, W)
$$

for $G$-representations $V$ and $W$. If $V$ is simple and $W$ semisimple, $[V, W]=[W, V]$ counts the multiplicity of $V$ in $W$.

Proposition 2.1. Let $\lambda, \mu, \nu$ be partitions. Then the Littlewood-Richardson coefficient $c_{\mu \nu}^{\lambda}$ computes the following multiplicities.

$$
\left[\operatorname{Res}_{\mathfrak{S}_{m} \times \mathfrak{S}_{n}}^{\mathfrak{S}_{m+n}} \mathfrak{S}_{m+n}(\lambda), \mathfrak{S}_{m}(\mu) \otimes \mathfrak{S}_{n}(\nu)\right]=c_{\mu \nu}^{\lambda}
$$

if $|\lambda|=m+n,|\mu|=m,|\nu|=n$.

$$
\left[\operatorname{Res}_{\mathrm{GL}_{m} \mathbb{Q} \times \mathrm{GL}_{n} \mathbb{Q}}^{\mathrm{GL}_{m+n}} \operatorname{GL}_{m+n}(\lambda), \mathrm{GL}_{m}(\mu) \otimes \mathrm{GL}_{n}(\nu)\right]=c_{\mu \nu}^{\lambda}
$$

if $\ell(\lambda) \leq m+n, \ell(\mu) \leq m, \ell(\nu) \leq n$.

$$
\left[\mathrm{GL}_{n}(\mu) \otimes \mathrm{GL}_{n}(\nu), \mathrm{GL}_{n}(\lambda)\right]=c_{\mu \nu}^{\lambda}
$$

if $\ell(\lambda), \ell(\mu), \ell(\nu) \leq n$.

The second proposition implies that all sums over partitions that appear in this paper are finite sums.

Proposition 2.2. The Littlewood-Richardson coefficient $c_{\mu \nu}^{\lambda}$ is zero unless

$$
|\mu|+|\nu|=|\lambda|
$$

and both $\mu$ and $\nu$ are subdiagrams of $\lambda$.

2.5. Some simple branching rules. The main tool of this paper will be the branching rules for rational representations. For the restrictions $\operatorname{Res}_{\mathrm{GL}_{n-1} \mathbb{Q}}^{\mathrm{GL}_{n} \mathbb{Q}} \mathrm{GL}_{n}\left(\lambda^{+}, \lambda^{-}\right)$ and $\operatorname{Res}_{\mathrm{Sp}_{2 n-2} \mathbb{Q}}^{\mathrm{Sp}_{2 n} \mathbb{Q}} \operatorname{Sp}_{2 n}(\lambda)$ there are some simple rules that can be found in GoodmanWallach [GW09, Thm 8.1.1, Thm 8.1.5]. To phrase these for the rational representations of the general linear groups, let $\lambda \in \mathbb{Z}^{n}$ with

$$
\lambda_{1}=\lambda_{1}^{+} \geq \lambda_{2}=\lambda_{2}^{+} \geq \cdots \geq \lambda_{n-1}=-\lambda_{2}^{-} \geq \lambda_{n}=-\lambda_{1}^{-}
$$

for a pair of partitions $\left(\lambda^{+}, \lambda^{-}\right)$with length $\ell\left(\lambda^{+}\right)+\ell\left(\lambda^{-}\right) \leq n$.

Theorem 2.3. The multiplicity

$$
\left[\operatorname{Res}_{\mathrm{GL}_{n-1} \mathbb{Q}}^{\mathrm{GL}_{n} \mathbb{Q}} \mathrm{GL}_{n}\left(\lambda^{+}, \lambda^{-}\right), \mathrm{GL}_{n-1}\left(\mu^{+}, \mu^{-}\right)\right]=1
$$

if and only if

$$
\lambda_{1} \geq \mu_{1} \geq \lambda_{2} \geq \mu_{2} \geq \cdots \geq \lambda_{n-1} \geq \mu_{n-1} \geq \lambda_{n} .
$$

Otherwise it is zero. 
Theorem 2.4. The multiplicity

$$
\left[\operatorname{Res}_{\mathrm{Sp}_{2 n-2} \mathbb{Q}}^{\mathrm{Sp}_{2 n} \mathbb{Q}} \operatorname{Sp}_{2 n}(\lambda), \operatorname{Sp}_{2 n-2}(\mu)\right]
$$

is nonzero if and only if

$$
\lambda_{i} \geq \mu_{i} \geq \lambda_{i+2}
$$

for all $1 \leq i \leq n-1$. ( $\lambda_{n+1}$ is always zero.)

In both cases, we obtain a corollary, which will prove useful later.

Corollary 2.5. If

$$
\left[\operatorname{Res}_{\mathrm{GL}_{n-m}}^{\mathrm{GL}_{n} \mathbb{Q}} \mathrm{GL}_{n}\left(\lambda^{+}, \lambda^{-}\right), \mathrm{GL}_{n-m}\left(\mu^{+}, \mu^{-}\right)\right] \neq 0
$$

then

$$
\ell\left(\mu^{+}\right)+\ell\left(\mu^{-}\right) \geq \ell\left(\lambda^{+}\right)+\ell\left(\lambda^{-}\right)-2 m .
$$

Proof. First assume $m=1$. Let $r^{+}=\ell\left(\lambda^{+}\right), r^{-}=\ell\left(\lambda^{-}\right)$. Then from Theorem 2.3, we know that

$$
\mu_{r^{+-1}}^{+} \geq \lambda_{r^{+}}^{+}>0>-\lambda_{r^{-}}^{-} \geq \mu_{r^{-}-1}^{-}
$$

This implies

$$
\ell\left(\mu^{+}\right)+\ell\left(\mu^{-}\right) \geq r^{+}-1+r^{-}-1=\ell\left(\lambda^{+}\right)+\ell\left(\lambda^{-}\right)-2
$$

and proves the assertion for $m=1$. For $m>1$ the corollary follows by induction.

Corollary 2.6. If

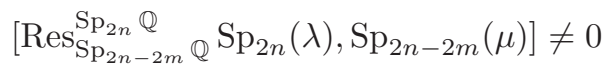

then

$$
\ell(\mu) \geq \ell(\lambda)-2 m
$$

Proof. For $m=1$ this follows from Theorem 2.4, because

$$
\mu_{r-2} \geq \lambda_{r}>0
$$

for $r=\ell(\lambda)$. For $m>1$ the corollary follows by induction.

For relatively small partitions $\lambda^{+}, \lambda^{-}, \lambda$, the multiplicities of some irreducible constituents of $\operatorname{Res}_{\mathrm{GL}_{m}+n}^{\mathrm{GL}_{m} \times \mathrm{GL}_{n} \mathbb{Q}} \mathrm{GL}_{n}\left(\lambda^{+}, \lambda^{-}\right)$and $\operatorname{Res}_{\mathrm{Sp}_{2 m}}^{\mathrm{Sp}_{2 m+2 n} \times \operatorname{Sp}_{2 n} \mathbb{Q}} \operatorname{Sp}_{2 n}(\lambda)$ can be expressed nicely in the stable branching rules. For the following results we quote Howe-Tan-Willenbring [HTW05, 2.2.1, 2.2.3].

Theorem 2.7. Let $m, n, p, q \in \mathbb{N}$ such that $p+q \leq \min (m, n)$. Let $\lambda^{+}, \mu^{+}, \nu^{+}$be partitions with at most $p$ rows and $\lambda^{-}, \mu^{-}, \nu^{-}$with at most $q$ rows. Then

$$
\begin{aligned}
{\left[\operatorname{Res}_{\mathrm{GL}_{m} \mathbb{Q} \times \mathrm{GL}_{n} \mathbb{Q}}^{\mathrm{GL}_{m+n}} \mathrm{GL}_{m+n}\left(\lambda^{+}, \lambda^{-}\right), \mathrm{GL}_{m}\left(\mu^{+}, \mu^{-}\right) \otimes \mathrm{GL}_{n}\left(\nu^{+}, \nu^{-}\right)\right] } \\
=\sum_{\gamma^{+}, \gamma^{-}, \delta} c_{\mu^{+} \nu^{+}}^{\gamma^{+}} c_{\mu^{-} \nu^{-}}^{\gamma^{-}} c_{\gamma^{+} \delta^{+}}^{\lambda^{+}} c_{\gamma^{-} \delta}^{\lambda^{-}}
\end{aligned}
$$


Theorem 2.8. Let $\lambda, \mu, \nu$ be partitions with at most $\min (m, n)$ rows. Then

$$
\left[\operatorname{Res}_{\mathrm{Sp}_{2 m} \mathbb{Q} \times \operatorname{Sp}_{2 n} \mathbb{Q}}^{\mathrm{Sp}_{2 m+2 n} \mathbb{Q}} \operatorname{Sp}_{2 m+2 n}(\lambda), \operatorname{Sp}_{2 m}(\mu) \otimes \operatorname{Sp}_{2 n}(\nu)\right]=\sum_{\gamma, \delta} c_{\mu \nu}^{\gamma} c_{\gamma(2 \delta)^{\prime}}^{\lambda}
$$

where $(2 \delta)^{\prime}$ is a partition with only even column lengths.

2.6. Modification rules for $\mathrm{GL}_{n} \mathbb{Q}$. In order to state the branching rules more generally, we need modification rules. To that effect we will use Koike and Tereda's theory of universal characters introduced in [KT87, Koi89].

Let

$$
\Lambda_{x}={\underset{\lim }{n}}_{n}\left[x_{1}, \ldots, x_{n}\right]^{\mathfrak{S}_{n}}
$$

denote the ring of symmetric functions and

$$
\Lambda_{x y}=\Lambda_{x} \otimes \Lambda_{y}
$$

The Schur functions

$$
\left\{s_{\lambda}(x)\right\}_{\lambda} \text { a partition }
$$

form a basis of the free abelian group $\Lambda_{x}$ and thus the tensor products

$$
\left\{s_{\lambda}(x) \otimes s_{\mu}(y)\right\}_{\lambda, \mu \text { partitions }}
$$

form a basis of $\Lambda_{x y}$. Koike [Koi89, Sec 2] defines a ring homomorphism

$$
\tilde{\pi}_{n}: \Lambda_{x y} \longrightarrow R\left(\mathrm{GL}_{n} \mathbb{Q}\right)
$$

to the representation ring $R\left(\mathrm{GL}_{n} \mathbb{Q}\right)$ of the rational representations of $\mathrm{GL}_{n} \mathbb{Q}$. We denote

$$
\operatorname{modGL}{ }_{n}\left(\lambda^{+}, \lambda^{-}\right)=\tilde{\pi}_{n}\left(s_{\lambda^{+}}(x) \otimes s_{\lambda^{-}}(y)\right) .
$$

The "mod" stands for modification and and the laws that govern these "modified representations" are known under the name modification rules.

The idea is that the ring structure of $\Lambda_{x y}$ controls the branching rules of the rational representations of the general linear group. By design

$$
\operatorname{modGL}_{n}\left(\lambda^{+}, \lambda^{-}\right)=\mathrm{GL}_{n}\left(\lambda^{+}, \lambda^{-}\right)
$$

if $\ell\left(\lambda^{+}\right)+\ell\left(\lambda^{-}\right) \leq n$. In general, $\operatorname{modGL}_{n}\left(\lambda^{+}, \lambda^{-}\right)$is zero or a virtual representation $\pm \mathrm{GL}_{n}\left(\mu^{+}, \mu^{-}\right)$for some partitions $\mu^{+}, \mu^{-}$with $\ell\left(\mu^{+}\right)+\ell\left(\mu^{-}\right) \leq n$. These two statements are precisely [Koi89, Prop 2.2].

Sam-Snowden-Weyman [SSW13, Sec 5.4] give the following combinatorial construction of the modification rules. A border strip is a skew Young diagram that does not contain a $2 \times 2$ square. Its length is the number of boxes it contains. Assume $\ell\left(\lambda^{+}\right)+\ell\left(\lambda^{-}\right)>n$. Let, if they exist, $R_{\lambda^{+}}$and $R_{\lambda^{-}}$be the connected border strips of length $\ell\left(\lambda^{+}\right)+\ell\left(\lambda^{-}\right)-n-1$ in $\lambda^{+}$and $\lambda^{-}$containing the first box in the last row, respectively. If $\lambda^{+} \backslash R_{\lambda^{+}}$and $\lambda^{-} \backslash R_{\lambda^{-}}$are both Young diagrams again then

$$
\operatorname{modGL}_{n}\left(\lambda^{+}, \lambda^{-}\right)=(-1)^{c\left(R_{\lambda^{+}}\right)+c\left(R_{\lambda^{-}}\right)-1} \cdot \operatorname{modGL}{ }_{n}\left(\lambda^{+} \backslash R_{\lambda^{+}}, \lambda^{-} \backslash R_{\lambda^{-}}\right),
$$


where $c(R)$ denotes the number of columns the skew diagram $R$ occupies. If $R_{\lambda^{+}}$ or $R_{\lambda^{-}}$do not exist or are empty, or $\lambda^{+} \backslash R_{\lambda^{+}}$or $\lambda^{-} \backslash R_{\lambda^{-}}$are not Young diagrams then

$$
\operatorname{modGL} L_{n}\left(\lambda^{+}, \lambda^{-}\right)=0 .
$$

In this construction

$$
\ell\left(\lambda^{+} \backslash R_{\lambda^{+}}\right)+\ell\left(\lambda^{-} \backslash R_{\lambda^{-}}\right)<\ell\left(\lambda^{+}\right)+\ell\left(\lambda^{-}\right) .
$$

Therefore it terminates after finitely many steps.

We reproduce [SSW13, Ex 5.17]. Let $n=3, \lambda^{+}=(4,3,2,2)$ and $\lambda^{-}=$ $(5,2,2,1,1)$. Then $\ell\left(\lambda^{+}\right)+\ell\left(\lambda^{-}\right)=9$ and the border strips of length $9-3-1=5$ are marked by bullet points in following diagrams:
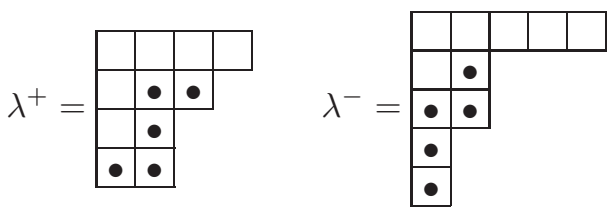

Because $R_{\lambda^{+}}$occupies 3 columns and $R_{\lambda^{-}}$occupies 4 columns,

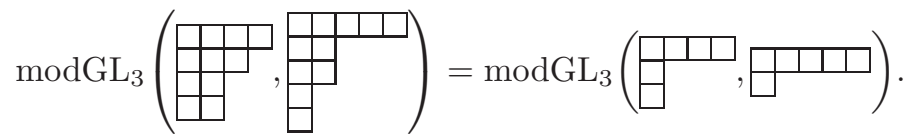

We again mark the border strips of length $5-3-1=1$ by bullet points:
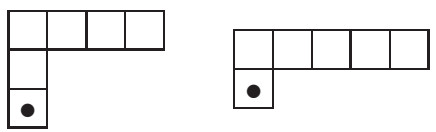

In the end we get

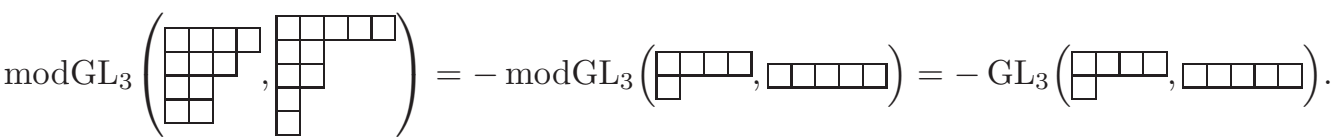

We summarize all that we will need in the later discussion in the following Proposition.

Proposition 2.9 (Modification rules for $\mathrm{GL}_{n} \mathbb{Q}$ ). Let $\lambda^{+}, \lambda^{-}$be partitions. Then:

(a) $\operatorname{modGL}_{n}\left(\lambda^{+}, \lambda^{-}\right)=\mathrm{GL}_{n}\left(\lambda^{+}, \lambda^{-}\right)$if $\ell\left(\lambda^{+}\right)+\ell\left(\lambda^{-}\right) \leq n$.

(b) $\operatorname{modGL}_{n}\left(\lambda^{+}, \lambda^{-}\right)$is zero or a virtual representation $\pm \mathrm{GL}_{n}\left(\mu^{+}, \mu^{-}\right)$for some partitions $\mu^{+}, \mu^{-}$with $\ell\left(\mu^{+}\right)+\ell\left(\mu^{-}\right) \leq n$.

(c) If $\operatorname{modGL} L_{n}\left(\lambda^{+}, \lambda^{-}\right)= \pm \operatorname{GL}_{n}\left(\mu^{+}, \mu^{-}\right)$then $\mu^{+}, \mu^{-}$are contained in $\lambda^{+}, \lambda^{-}$, respectively.

2.7. Modification rules for $\mathrm{Sp}_{2 n} \mathbb{Q}$. Koike-Tereda [KT87, Sec 2.1] denote by

$$
\left\{\chi_{\mathrm{Sp}}(\lambda)(x)\right\}_{\lambda} \text { a partition }
$$

another basis of $\Lambda_{x}$. They define in [KT87, Sec 2.2] a ring homomorphism

$$
\pi_{\mathrm{Sp}_{2 n}}: \Lambda_{x} \longrightarrow R\left(\operatorname{Sp}_{2 n} \mathbb{Q}\right)
$$


to the representation $\operatorname{ring} R\left(\mathrm{Sp}_{2 n} \mathbb{Q}\right)$ of the rational representations of $\mathrm{Sp}_{2 n} \mathbb{Q}$. We denote

$$
\operatorname{modSp} \operatorname{Sp}_{2 n}(\lambda)=\pi_{\mathrm{Sp}_{2 n}}\left(\chi_{\mathrm{Sp}}(\lambda)(x)\right) .
$$

Similar to the modification rules of the general linear group

$$
\operatorname{modSp} p_{2 n}(\lambda)=\operatorname{Sp}_{2 n}(\lambda)
$$

if $\ell(\lambda) \leq n$ and otherwise $\operatorname{modSp}_{2 n}(\lambda)$ is zero or a virtual representation of the form $\pm \operatorname{Sp}_{2 n}(\mu)$ for some partition $\mu$ with $\ell(\mu) \leq n$. Koike-Tereda prove this in [KT87, Prop 2.2.1(1)+Prop 2.4.1(ii)].

Sam-Snowden-Weyman [SSW13, Sec 3.4] give the following combinatorial construction of the modification rules. Assume $\ell(\lambda)>n$. Let, if it exists, $R_{\lambda}$ be the connected border strip of length $2(\ell(\lambda)-n-1)$ in $\lambda$ containing the first box in the last row. If $\lambda \backslash R_{\lambda}$ is a Young diagram again then

$$
\operatorname{modSp} p_{2 n}(\lambda)=(-1)^{c\left(R_{\lambda}\right)} \cdot \operatorname{modSp}{ }_{2 n}\left(\lambda \backslash R_{\lambda}\right) .
$$

If $R_{\lambda}$ does not exist or is empty, or $\lambda \backslash R_{\lambda}$ is not a Young diagram then

$$
\operatorname{modSp} \sin _{2 n}(\lambda)=0 \text {. }
$$

Again

$$
\ell\left(\lambda \backslash R_{\lambda}\right)<\ell(\lambda)
$$

implies that this procedure terminates after finitely many steps.

The following example is [SSW13, Ex 3.20]. Let $n=2$ and consider the partition $\lambda=(6,5,4,4,3,3,2)$. We give the border strips of all steps in the following picture:
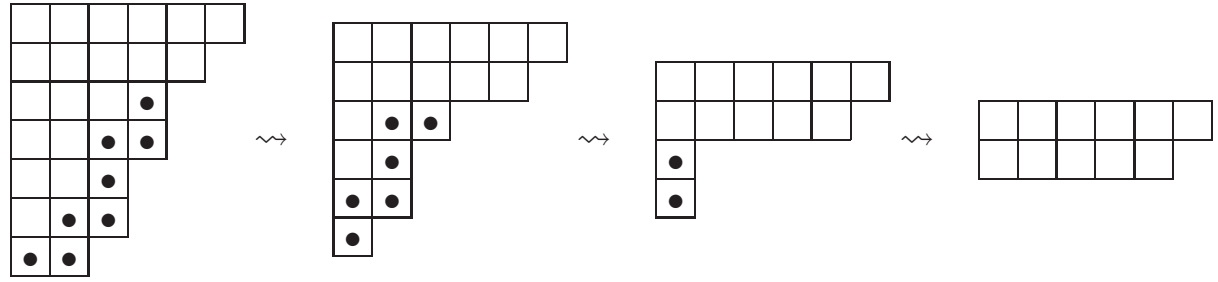

Therefore

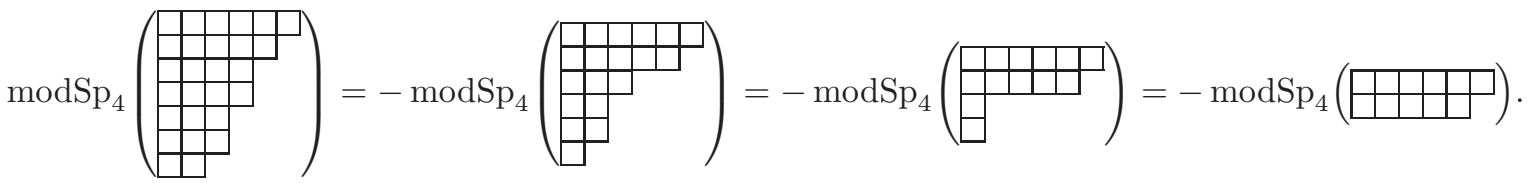

We summarize all that we will need in the later discussion in the following Proposition.

Proposition 2.10 (Modification rules for $\mathrm{Sp}_{2 n} \mathbb{Q}$ ). Let $\lambda$ be a partition. Then:

(a) $\operatorname{modSp} \sin _{2 n}(\lambda)=\operatorname{Sp}_{2 n}(\lambda)$ if $\ell(\lambda) \leq n$.

(b) $\operatorname{modSp}_{2 n}(\lambda)$ is zero or a virtual representation $\pm \operatorname{Sp}_{2 n}(\mu)$ for some partition $\mu$ with $\ell(\mu) \leq n$.

(c) If $\operatorname{modSp} \sin _{2 n}(\lambda)= \pm \operatorname{Sp}_{2 n}(\mu)$ then $\mu$ is contained in $\lambda$. 
2.8. Branching rules. The main technical tool of this paper will be branching rules of rational representations. We will need formulas for inner and outer tensor products and a stability statement for plethysms. These are corollaries of the modification rules.

Theorem 2.11 (Koike [Koi89, Thm 2.4]). Let $\mu^{+}, \mu^{-}, \nu^{+}, \nu^{-}$with $\ell\left(\mu^{+}\right)+\ell\left(\mu^{-}\right), \ell\left(\nu^{+}\right)+$ $\ell\left(\nu^{-}\right) \leq n$. Then

$$
\begin{aligned}
& \mathrm{GL}_{n}\left(\mu^{+}, \mu^{-}\right) \otimes \mathrm{GL}_{n}\left(\nu^{+}, \nu^{-}\right) \cong \\
& \bigoplus_{\lambda^{+}, \lambda^{-}} \operatorname{modGL} L_{n}\left(\lambda^{+}, \lambda^{-}\right)^{\oplus} \sum_{\alpha^{+}, \alpha^{-}, \beta^{+}, \beta^{-, \gamma, \delta}} c_{\alpha+\beta^{+}}^{\lambda^{+}} c_{\alpha+\gamma}^{\mu^{+}} c_{\beta+\delta}^{\nu^{+}} c_{\alpha-\beta^{-}}^{\lambda^{-}} c_{\alpha^{-} \delta^{-}}^{\mu_{\beta^{-}-\gamma}^{\nu^{-}}} .
\end{aligned}
$$

Theorem 2.12 (Koike [Koi89, Thm 3.1]). Let $\mu, \nu$ with $\ell(\mu), \ell(\nu) \leq n$. Then

$$
\operatorname{Sp}_{2 n}(\mu) \otimes \operatorname{Sp}_{2 n}(\nu) \cong \bigoplus_{\lambda} \operatorname{modSp} \operatorname{Sp}_{2 n}(\lambda)^{\oplus \sum_{\alpha, \beta, \gamma} c_{\alpha \beta}^{\lambda} c_{\alpha \gamma}^{\mu} c_{\beta \gamma}^{\nu}}
$$

Theorem 2.13 (Koike [Koi89, Prop 2.6]). Let $\lambda^{+}, \lambda^{-}$with $\ell\left(\lambda^{+}\right)+\ell\left(\lambda^{-}\right) \leq m+n$.

Then

$$
\begin{aligned}
& \operatorname{Res}_{\mathrm{GL}_{m} \mathbb{Q} \times \mathrm{GL}_{n} \mathbb{Q}}^{\mathrm{GL}_{m+n} \mathbb{Q}} \mathrm{GL}_{m+n}\left(\lambda^{+}, \lambda^{-}\right) \cong
\end{aligned}
$$

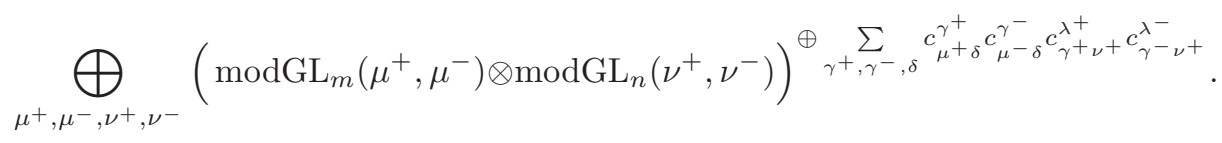

Theorem 2.14. Let $\lambda$ be a partition with $\ell(\lambda) \leq m+n$. Then

$$
\operatorname{Res}_{\operatorname{Sp}_{2 m} \mathbb{Q} \times \operatorname{Sp}_{2 n} \mathbb{Q}}^{\operatorname{Sp}_{2 m+2 n} \mathbb{Q}} \operatorname{Sp}_{2 m+2 n}(\lambda) \cong \bigoplus_{\mu, \nu}\left(\operatorname{modSp}_{2 m}(\mu) \otimes \operatorname{modSp}_{2 n}(\nu)\right)^{\oplus \sum_{\gamma, \delta} c_{\mu \nu}^{\gamma} c_{\gamma(2 \delta)^{\prime}}^{\lambda}} .
$$

Proof. In the philosophy of the proof of [Koi89, Prop 2.6], we consider two variable sets $x, y$ and the natural embedding

$$
\Lambda_{x \cup y} \longrightarrow \Lambda_{x} \otimes \Lambda_{y}
$$

where $x \cup y$ is the union of the variable sets $x$ and $y$. Then there is a unique way to write

$$
\chi_{\mathrm{Sp}}(\lambda)(x \cup y)=\sum m_{\mu \nu}^{\lambda} \chi_{\mathrm{Sp}}(\mu)(x) \otimes \chi_{\mathrm{Sp}}(\nu)(y) .
$$

Let $N \geq \max (\ell(\lambda), \ell(\mu)+\ell(\nu))$. Consider the following commutative diagram.

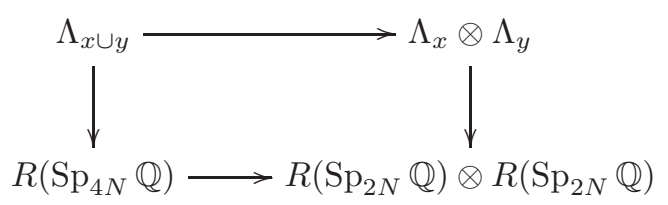

Then the stable branching rule Theorem 2.8 and Proposition 2.10(a) imply

$$
m_{\mu \nu}^{\lambda}=\sum_{\gamma, \delta} c_{\mu \nu}^{\gamma} c_{\gamma(2 \delta)^{\prime}}^{\lambda}
$$


Then the following commutative diagram proves the assertion.

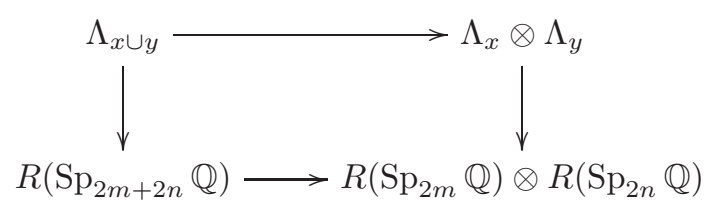

In the last paragraph of [Koi89, Sec 2] plethysms in the universal character ring $\Lambda_{x y}$ are introduced. That is if $\lambda, \mu^{+}, \mu^{-}$are partitions then there is an element $s_{\lambda}(x) \circ\left(s_{\mu^{+}}(x) \otimes s_{\mu^{-}}(y)\right) \in \Lambda_{x y}$ such that

$$
\tilde{\pi}_{n}\left(s_{\lambda}(x) \circ\left(s_{\mu^{+}}(x) \otimes s_{\mu^{-}}(y)\right)\right)=\mathrm{GL}_{n}\left(\mu^{+}, \mu^{-}\right)^{\otimes|\lambda|} \underset{\mathbb{Q} \mathfrak{S}_{|\lambda|}}{\otimes} \mathfrak{S}_{|\lambda|}(\lambda)
$$

for all $n \geq \ell\left(\mu^{+}\right)+\ell\left(\mu^{-}\right)$. We need the following consequence.

Proposition 2.15. Let $\lambda^{+}, \lambda^{-}$be partitions and $k \in \mathbb{N}$ then there is a large $N \in \mathbb{N}$ and fixed coefficients $m_{\mu^{+} \mu^{-}}$such that

$$
\bigwedge^{k} \mathrm{GL}_{n}\left(\lambda^{+}, \lambda^{-}\right) \cong \bigoplus_{\mu^{+}, \mu^{-}} \operatorname{GL}_{n}\left(\mu^{+}, \mu^{-}\right)^{\oplus m_{\mu^{+} \mu^{-}}}
$$

for all $n \geq N$.

Proof. Let us write

$$
s_{\left(1^{k}\right)}(x) \circ\left(s_{\lambda^{+}}(x) \otimes s_{\lambda^{-}}(y)\right)=\sum_{\mu^{+}, \mu^{-}} m_{\mu^{+} \mu^{-}} \cdot s_{\mu^{+}}(x) \otimes s_{\mu^{-}}(y)
$$

in $\Lambda_{x y}$. This is a finite sum and let $N$ be the maximal value $\ell\left(\mu^{+}\right)+\ell\left(\mu^{-}\right)$of those pairs $\left(\mu^{+}, \mu^{-}\right)$for which $m_{\mu^{+} \mu^{-}} \neq 0$. Then applying $\tilde{\pi}_{n}$ gives

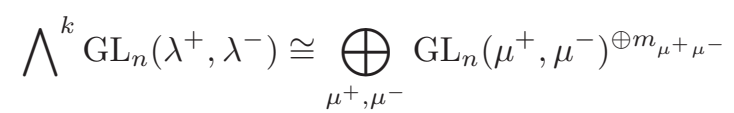

for all $n \geq N$ as asserted.

The analogous statement for $\operatorname{Sp}_{2 n} \mathbb{Q}$ can be found in [CF13] or can be proved analogously.

Proposition 2.16 (Church-Farb [CF13, Thm 3.1]). Let $\lambda$ be a partition and $k \in \mathbb{N}$ then there is a large $N \in \mathbb{N}$ and fixed coefficients $m_{\mu}$ such that

$$
\bigwedge^{k} \operatorname{Sp}_{n}(\lambda) \cong \bigoplus_{\mu} \operatorname{Sp}_{n}(\mu)^{\oplus m_{\mu}}
$$

for all $n \geq N$.

The following corollaries are needed in Section 3.

Corollary 2.17. Let $\lambda^{+}, \lambda^{-}$be partitions with $\ell\left(\lambda^{+}\right)+\ell\left(\lambda^{-}\right) \leq n$ and let $\mu^{+}, \mu^{-}$ be partitions with $\ell\left(\mu^{+}\right)+\ell\left(\mu^{-}\right) \leq m$. Assume further $\left|\lambda^{+}\right|+\left|\lambda^{-}\right| \leq\left|\mu^{+}\right|+\left|\mu^{-}\right|$, 
then

$$
\begin{aligned}
& {\left[\operatorname{Res}_{\mathrm{GL}_{m} \mathbb{Q} \times \mathrm{GL}_{n-m} \mathbb{Q}}^{\mathrm{GL}_{n} \mathbb{Q}} \operatorname{GL}_{n}\left(\lambda^{+}, \lambda^{-}\right), \mathrm{GL}_{m}\left(\mu^{+}, \mu^{-}\right) \otimes \mathrm{GL}_{n-m}\left(\nu^{+}, \nu^{-}\right)\right]} \\
& = \begin{cases}1 & \text { if } \mu^{+}=\lambda^{+}, \mu^{-}=\lambda^{-} \text {and } \nu^{+}=\nu^{-}=\emptyset \\
0 & \text { otherwise. }\end{cases}
\end{aligned}
$$

In particular, if $\left|\lambda^{+}\right|+\left|\lambda^{-}\right|<\left|\mu^{+}\right|+\left|\mu^{-}\right|$then

$$
\operatorname{Hom}_{\mathrm{GL}_{m} \mathbb{Q}}\left(\mathrm{GL}_{m}\left(\mu^{+}, \mu^{-}\right), \operatorname{Res}_{\mathrm{GL}_{m} \mathbb{Q}}^{\mathrm{GL}_{n} \mathbb{Q}} \mathrm{GL}_{n}\left(\lambda^{+}, \lambda^{-}\right)\right)=0 .
$$

Similarly if $\ell\left(\lambda^{+}\right)<\ell\left(\mu^{+}\right)$or $\ell\left(\lambda^{-}\right)<\ell\left(\mu^{-}\right)$then

$$
\operatorname{Hom}_{\mathrm{GL}_{m} \mathbb{Q}}\left(\mathrm{GL}_{m}\left(\mu^{+}, \mu^{-}\right), \operatorname{Res}_{\mathrm{GL}_{m}}^{\mathrm{GL}_{n} \mathbb{Q}} \mathrm{GL}_{n}\left(\lambda^{+}, \lambda^{-}\right)\right)=0 .
$$

Proof. From Proposition 2.9(c), we know that $\left|\operatorname{modGL}_{m}\left(\eta^{+}, \eta^{-}\right)\right| \leq\left|\eta^{+}\right|+\left|\eta^{-}\right|$. Thus if $\operatorname{modGL} L_{m}\left(\eta^{+}, \eta^{-}\right)=\left(\mu^{+}, \mu^{-}\right)$, we also know $\left|\eta^{+}\right|+\left|\eta^{-}\right| \geq\left|\lambda^{+}\right|+\left|\lambda^{-}\right|$. For such $\eta^{+}, \eta^{-}$we can calculate the multiplicity from Theorem 2.13.

$c_{\eta^{+} \nu^{+}}^{\gamma^{+}} c_{\eta^{-} \nu^{-}}^{\gamma^{-}} c_{\gamma^{+} \delta}^{\lambda^{+}} c_{\gamma^{-} \delta}^{\lambda^{-}}= \begin{cases}1 & \text { if } \eta^{+}=\gamma^{+}=\lambda^{+}, \eta^{-}=\gamma^{-}=\lambda^{-} \text {and } \nu^{+}=\nu^{-}=\delta=\emptyset \\ 0 & \text { otherwise. }\end{cases}$

Therefore the only constituent

$$
\operatorname{modGL} L_{m}\left(\eta^{+}, \eta^{-}\right) \otimes \operatorname{modGL} L_{n-m}\left(\nu^{+}, \nu^{-}\right)
$$

in $\operatorname{Res}_{\mathrm{GL}_{m}}^{\mathrm{GL}_{m+n} \times \mathrm{GL}_{n} \mathbb{Q}} \mathbb{Q} \mathrm{GL}_{m+n}$ with $\left|\eta^{+}\right|+\left|\eta^{-}\right| \geq\left|\lambda^{+}\right|+\left|\lambda^{-}\right|$is

$$
\operatorname{modGL} L_{m}\left(\mu^{+}, \mu^{-}\right) \otimes \operatorname{modGL} L_{n-m}(\emptyset, \emptyset)=\operatorname{GL}_{m}\left(\mu^{+}, \mu^{-}\right) \otimes \mathrm{GL}_{n-m}(\emptyset) .
$$

Corollary 2.18. Let $\lambda$ be a partition with $\ell(\lambda) \leq n$ and let $\mu$ be a partition with $\ell(\mu) \leq m$. Assume further $|\lambda| \leq|\mu|$, then

$$
\left[\operatorname{Res}_{\operatorname{Sp}_{2 m}}^{\operatorname{Sp}_{2 n} \mathbb{Q} \times \operatorname{Sp}_{2 n-2 m}} \mathbb{Q} \operatorname{Sp}_{2 n}(\lambda), \operatorname{Sp}_{2 m}(\mu) \otimes \operatorname{Sp}_{2 n-2 m}(\nu)\right]= \begin{cases}1 & \text { if } \mu=\lambda \text { and } \nu=\emptyset \\ 0 & \text { otherwise. }\end{cases}
$$

In particular, if $|\lambda|<|\mu|$ then

$$
\operatorname{Hom}_{\mathrm{Sp}_{2 m} \mathbb{Q}}\left(\operatorname{Sp}_{2 m}(\mu), \operatorname{Res}_{\mathrm{Sp}_{2 m} \mathbb{Q}}^{\mathrm{Sp}_{2 n}} \mathbb{Q} \operatorname{Sp}_{2 n}(\lambda)\right)=0 .
$$

Proof. Analogous to Corollary 2.17 we calculate the multiplicity from Theorem 2.14 for $|\eta| \geq|\lambda|$.

$$
c_{\eta \nu}^{\gamma} c_{\gamma(2 \delta)^{\prime}}^{\lambda}= \begin{cases}1 & \text { if } \eta=\gamma=\lambda \text { and } \nu=\delta=\emptyset \\ 0 & \text { otherwise. }\end{cases}
$$

2.9. Restriction to $\mathrm{GL}_{n} \mathbb{Z}$ and $\mathrm{Sp}_{2 n} \mathbb{Z}$. We will later need to understand the restrictions $\operatorname{Res}_{\mathrm{GL}_{n} \mathbb{Z}}^{\mathrm{GL}_{n} \mathbb{Q}} \mathrm{GL}_{n}\left(\lambda^{+}, \lambda^{-}\right)$and $\operatorname{Res}_{\mathrm{Sp}_{2 n}}^{\mathrm{Sp}_{2 n}} \mathbb{\mathbb { Q }} \operatorname{Sp}_{n}(\lambda)$. The information we need is provided by Borel in the context of his density theorem:

Theorem 2.19 (Borel [Bor60, Prop 3.2]). Let $G$ be a simple non-compact connected real Lie group and $V$ a finite dimensional irreducible $G$-representation. Let $H$ be a subgroup of $G$ such that for every neighborhood $U$ of the identity in $G$ and every 
$g \in G$ there exists an integer $n>0$ with $g^{n} \in U \cdot H \cdot U$. Then $V$ is an irreducible $H$-representation.

This theorem directly applies to the symplectic groups $G=\mathrm{Sp}_{2 n} \mathbb{R}$ and $H=$ $\mathrm{Sp}_{2 n} \mathbb{Z}$ and can also be transferred to stating that

$$
\operatorname{Res}_{\mathrm{Sp}_{2 n}}^{\mathrm{Sp}_{2 n}} \mathbb{\mathbb { Q }} \mathrm{Sp}_{2 n}(\lambda)
$$

is an irreducible $\operatorname{Sp}_{2 n} \mathbb{Z}$-representation for all partitions $\lambda$ of length $\ell(\lambda) \leq n$. Furthermore because $H$ is Zariski dense in $G$ (which is the main result of [Bor60]) all of these $\mathrm{Sp}_{2 n} \mathbb{Z}$-representations are pairwise nonisomorphic.

To understand the situation for the general linear group, we need to take a look at the representation theory of the special linear group. Essentially, the difference between the rational representation theory of these two groups is the determinant representation, which restricts to the trivial $\mathrm{SL}_{n} \mathbb{Q}$-representation. In fact, all irreducible polynomial (which is the same as rational) $\mathrm{SL}_{n} \mathbb{Q}$-representations are given by and are uniquely (up to isomorphism) determined by

$$
\operatorname{Res}_{\mathrm{SL}_{n}}^{\mathrm{GL}_{n} \mathbb{Q}} \underset{\mathbb{Q}}{\mathrm{GL}_{n}}(\lambda)=V_{n}^{\otimes r} \underset{\mathbb{Q} \mathfrak{S}_{r}}{\otimes} \mathfrak{S}_{r}(\lambda)
$$

for some partition $\lambda$ with length $\ell(\lambda) \leq n-1$. In general, the restriction is not much harder. Every irreducible rational GL $\mathbb{Q}_{n}$-representation can be written as a tensor product

$$
\mathrm{GL}_{n}\left(\lambda^{+}, \lambda^{-}\right) \cong \mathrm{GL}_{n}(\lambda) \otimes D_{k}
$$

for a uniquely determined partition $\lambda$ with length $\ell(\lambda) \leq n-1$ and $k=\lambda_{1}^{-} \in \mathbb{Z}$ or $k=-\lambda_{n}^{+} \in \mathbb{Z}$ if $\lambda^{-}$is empty. Because

$$
\operatorname{Res}_{\mathrm{SL}_{n} \mathbb{Q}}^{\mathrm{GL}} D_{k}
$$

is the trivial representation, we have completely described the restriction of irreducible rational $\mathrm{GL}_{n} \mathbb{Q}$-representations to $\mathrm{SL}_{n} \mathbb{Q}$.

Theorem 2.19 is now applicable to $G=\mathrm{SL}_{n} \mathbb{R}$ and $H=\mathrm{SL}_{n} \mathbb{Z}$ and can be transferred to the statement that

$$
\operatorname{Res}_{\mathrm{SL}_{n} \mathbb{Z}}^{\mathrm{SL}_{n} \mathbb{Q}} \mathrm{SL}_{n}(\lambda)
$$

is an irreducible $\mathrm{SL}_{n} \mathbb{Z}$-representation for all partitions $\lambda$ of length $\ell(\lambda) \leq n-1$. Again all these $\mathrm{SL}_{n} \mathbb{Z}$-representations are pairwise nonisomorphic.

For the general linear group this implies that

$$
\operatorname{Res}_{\mathrm{GL}_{n} \mathbb{Z}}^{\mathrm{GL}_{n}} \mathbb{Q} \mathrm{GL}_{n}\left(\lambda^{+}, \lambda^{-}\right)
$$

is an irreducible $\mathrm{GL}_{n} \mathbb{Z}$-representation. Note that

$$
\operatorname{Res}_{\mathrm{GL}_{n} \mathbb{Z}}^{\mathrm{GL}_{n} \mathbb{Q}} D_{k} \cong \operatorname{Res}_{\mathrm{GL}_{n} \mathbb{Z}}^{\mathrm{GL}_{n} \mathbb{Q}} D_{k+2}
$$

and thus all restrictions of irreducible rational $\mathrm{GL}_{n} \mathbb{Q}$-representations to $\mathrm{GL}_{n} \mathbb{Z}$ have the form

$$
\operatorname{Res}_{\mathrm{GL}_{n} \mathbb{Z}}^{\mathrm{GL}_{n} \mathbb{Q}}\left(\mathrm{GL}_{n}(\lambda) \otimes D_{k}\right)
$$


for some partition $\lambda=\left(\lambda_{1}, \ldots, \lambda_{r}\right)$ of length $r=\ell(\lambda) \leq n-1$ and $k \in\{0,-1\}$. Such a $\mathrm{GL}_{n} \mathbb{Q}$-representation has the highest weight

$$
\left(\lambda_{1}+k\right) L_{1}+\cdots+\left(\lambda_{r}+k\right) L_{r}+k L_{r+1}+\cdots+k L_{n}
$$

so it is exactly one

$$
\mathrm{GL}_{n}\left(\lambda^{+}, \lambda^{-}\right)
$$

such that $\ell\left(\lambda^{+}\right) \leq n-1$ and $\lambda^{-}$is contained in $\left(1^{n}\right)$.

Assume

$$
\operatorname{Res}_{\mathrm{GL}_{n} \mathbb{Z}}^{\mathrm{GL}}\left(\mathrm{GL}_{n}(\lambda) \otimes D_{k}\right) \cong \operatorname{Res}_{\mathrm{GL}_{n} \mathbb{Z}}^{\mathrm{GL}_{n}} \mathbb{Q}\left(\mathrm{GL}_{n}\left(\lambda^{\prime}\right) \otimes D_{k^{\prime}}\right)
$$

are isomorphic, then by restriction to $\mathrm{SL}_{n} \mathbb{Z}$, we see that $\lambda=\lambda^{\prime}$. By an argument communicated to the author by David Speyer we can prove that

$$
\operatorname{Res}_{\mathrm{GL}_{n} \mathbb{Z}}^{\mathrm{GL}_{n} \mathbb{Q}}\left(\mathrm{GL}_{n}(\lambda) \otimes D_{-1}\right) \not \operatorname{Res}_{\mathrm{GL}_{n} \mathbb{Z}}^{\mathrm{GL}_{n}} \mathbb{G L}_{n}(\lambda) \text {. }
$$

The argument goes as follows. Let $\rho$ denote the representation of $\operatorname{GL}_{n} \mathbb{Z}$ on $\operatorname{GL}_{n}(\lambda)$. If we assume there exists an isomorphism then its characters must coincide:

$$
(\operatorname{det} g)^{-1} \cdot \operatorname{tr} \rho(g)=\operatorname{tr} \rho(g)
$$

Thus all $g \in \mathrm{GL}_{n} \mathbb{Z}$ with negative determinant must have vanishing value of the character of $\rho$. The character can be described by the (complex) eigenvalues $\alpha_{1}, \ldots, \alpha_{n}$ of $g$ as

$$
\operatorname{tr} \rho(g)=s_{\lambda}\left(\alpha_{1}, \ldots, \alpha_{n}\right)
$$

where $s_{\lambda}$ is the Schur polynomial, which is symmetric and homogeneous of degree $|\lambda|$. Therefore $s_{\lambda}$ can not be divisible by the inhomogeneous polynomial $1+x_{1} \cdots x_{n}$. Let us write $s_{\lambda}$ as a polynomial $p$ in the elementary symmetric polynomials $e_{1}, \ldots, e_{n}$. Then the previous statement is precisely that $p$ is not divisible by $1+e_{n}$. Because $\mathbb{Z}^{n}$ is Zariski closed in $\mathbb{Q}^{n}$, there are integers $\left(f_{1}, \ldots, f_{n}\right)$ such that $p\left(f_{1}, \ldots, f_{n}\right) \neq 0$ but $1+f_{n}=0$. Let

$$
g=\left(\begin{array}{cccc}
0 & \cdots & 0 & (-1)^{n+1} f_{n} \\
1 & \ddots & \vdots & (-1)^{n} f_{n-1} \\
& \ddots & 0 & \vdots \\
0 & & 1 & f_{1}
\end{array}\right) \in \mathrm{GL}_{n} \mathbb{Z}
$$

be the companion matrix to the characteristic polynomial

$$
x^{n}-f_{1} x^{n-1}+\cdots+(-1)^{n} f_{n} .
$$

Say $\alpha_{1}, \ldots, \alpha_{n}$ are the (complex) roots of the characteristic polynomial, that are the eigenvalues of $g$, then

$$
\operatorname{tr} \rho(g)=s_{\lambda}\left(\alpha_{1}, \ldots \alpha_{n}\right)=p\left(f_{1}, \ldots, f_{n}\right) \neq 0
$$

even though the determinant $\operatorname{det} g=f_{n}=-1$. Contradiction. 


\section{Representation stability For General Linear Groups AND SYMPLECTIC GROUPS}

When Church-Ellenberg-Farb [CEF15] study representation stable sequences of representations of the symmetric groups, they consider modules over the category $\mathrm{FI}$ of finite sets and injections. When we want to generalize their work to the general linear groups and symplectic groups, the obvious generalizations of FI would be VI and SI, the category of finite dimensional vector spaces and injections and the category of symplectic vector spaces and (injective) isometries. For the symplectic groups this turns out to be correct but for the general linear groups we need a different notion. The following definition of VIC, which stands for vector spaces with injections and complements, was related to representation stability by Putman-Sam [PS14].

\subsection{VIC and SI.}

Definition 3.1. Fix a commutative ring $R$. Let $\mathrm{VIC}_{R}$ be the category whose objects are finite rank free modules over $R$ and its morphisms are given by a monomorphism together with a free complement of the image. That is

$$
\operatorname{Hom}_{\mathrm{VIC}}(V, W)=\{(f, C) \mid f: V \longleftrightarrow W, \operatorname{im} f \oplus C=W, C \text { free }\} .
$$

The composition is given by

$$
(g, D) \circ(f, C)=(g \circ f, D \oplus g(C)) .
$$

Let $\mathrm{SI}_{R}$ be the category whose objects are finitely generated symplectic free modules over $R$ and its morphisms are given by isometries. Here a free module of rank $2 n$ together with a bilinear form $\langle,\rangle_{\mathrm{Sp}}$ is symplectic if there is a basis $\left\{e_{1}, e_{1}^{\prime}, \ldots, e_{n}, e_{n}^{\prime}\right\}$ such that $\left\langle e_{i}, e_{j}\right\rangle_{\mathrm{Sp}}=\left\langle e_{i}^{\prime}, e_{j}^{\prime}\right\rangle_{\mathrm{Sp}}=0$ and $\left\langle e_{i}, e_{j}^{\prime}\right\rangle_{\mathrm{Sp}}=-\left\langle e_{i}^{\prime}, e_{j}\right\rangle_{\mathrm{Sp}}=\delta_{i j}$. Isometries are always injective but not necessarily bijective.

The property by which we chose VIC and SI for our purpose is pointed out by the following remark.

Remark 3.2. A skeleton of $\mathrm{VIC}_{R}$ is given by the full subcategory on the objects $\left\{R^{n}\right\}_{n \in \mathbb{N}}$ and

$$
\operatorname{Hom}_{\mathrm{VIC}_{R}}\left(R^{m}, R^{n}\right) \cong \begin{cases}\mathrm{GL}_{n} R / \mathrm{GL}_{n-m} R & \text { if } n \geq m, \\ \emptyset & \text { otherwise. }\end{cases}
$$

Composition is given by group multiplication:

$$
\begin{aligned}
\mathrm{GL}_{n} R / \mathrm{GL}_{n-m} R \times \mathrm{GL}_{m} R / \mathrm{GL}_{m-l} R & \longrightarrow \mathrm{GL}_{n} R / \mathrm{GL}_{n-l} R \\
\left(g \mathrm{GL}_{n-m} R, h \mathrm{GL}_{m-l} R\right) & \longmapsto g h \mathrm{GL}_{n-l} R
\end{aligned}
$$


Similarly a skeleton of $\mathrm{SI}_{R}$ is given by the full subcategory on the objects $\left\{R^{2 n}\right\}_{n \in \mathbb{N}}$ and

$$
\operatorname{Hom}_{\mathrm{SI}_{R}}\left(R^{2 m}, R^{2 n}\right) \cong \begin{cases}\mathrm{Sp}_{2 n} R / \mathrm{Sp}_{2 n-2 m} R & \text { if } n \geq m \\ \emptyset & \text { otherwise. }\end{cases}
$$

Composition is also given by group multiplication.

3.2. VIC- and SI-modules. Let us fix a commutative ring $R$.

Definition 3.3. Let $\mathcal{C}$ be a category, then $\mathcal{C}$-modules are functors from $\mathcal{C}$ to the category $\mathbb{Q}$-mod of vector spaces over $\mathbb{Q}$. Note that for $\mathcal{C}=\mathrm{VIC}_{R}$ and $\mathcal{C}=\mathrm{SI}_{R}$, it is enough to consider the effect of such a functor $V$ on the skeleton which is indexed by the natural numbers. By these means we will write $V_{n}$ for image of $R^{n}$ or $R^{2 n}$ under $V$, respectively. Furthermore we will denote the image of the standard embedding $R^{n} \rightarrow R^{n+1}$ and $R^{2 n} \rightarrow R^{2 n+2}$ by $\phi_{n}: V_{n} \rightarrow V_{n+1}$.

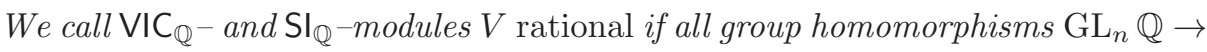
$\mathrm{GL}\left(V_{n}\right)$ and $\mathrm{Sp}_{2 n} \mathbb{Q} \rightarrow \mathrm{GL}\left(V_{n}\right)$ are rational.

We will consider representable $\mathcal{C}$-modules as free.

Definition 3.4. Denote the representable functors $\mathbb{Q}\left[\operatorname{Hom}_{\mathrm{VIC}_{R}}\left(R^{m},-\right)\right]$ and $\mathbb{Q}\left[\operatorname{Hom}_{\mathrm{SI}_{R}}\left(R^{2 m},-\right)\right]$ uniformly by $M(m)$.

We call $\mathrm{VIC}_{R^{-}}$and $\mathrm{SI}_{R}$-modules $V$ generated in ranks $\leq m$ if there is a surjection

$$
\bigoplus_{i \in I} M\left(m_{i}\right) \longrightarrow V
$$

where $m_{i} \leq m$ for all $i \in I$. (The index set $I$ is allowed to be infinite.)

$W e$ say $V$ is generated in finite rank if it is generated in ranks $\leq m$ for some $m \in \mathbb{N}$.

There is a good reason to consider $M(m)$ free. By the Yoneda Lemma, a homomorphism

$$
M(m) \longrightarrow V
$$

for some $\mathcal{C}$-module $V$ is determined by the image of id $\in M(m)_{m}$ in $V_{m}$. Also if

$$
\bigoplus_{i \in I} M\left(m_{i}\right) \longrightarrow V
$$

the smallest submodule of $V$ that contains the images of id $\in M\left(m_{i}\right)_{m_{i}}$ is $V$ itself. Thus $V$ is generated by those images, which all lie in ranks $\leq m$.

We will need the following propositions later to provide sequences of representations with a functorial structure. They are the natural generalizations of Church-Ellenberg-Farb [CEF15, Rem 3.3.1] to $\mathrm{VIC}_{R}$ and $\mathrm{SI}_{R}$. Randal-WilliamsWahl [RWW15, Prop 4.2] prove it in a more general setup.

Proposition 3.5. Let $\left\{V_{n}\right\}_{n \in \mathbb{N}}$ be a sequence of $\mathrm{GL}_{n} R$-representations and let $\phi_{n}: V_{n} \rightarrow V_{n+1}$ be $\mathrm{GL}_{n} R$-equivariant maps. Then $\mathrm{GL}_{n-m} R$ acts trivially on the image of $V_{m}$ in $V_{n}$ if and only if there is a $\mathrm{VIC}_{R}$-module $V$ with $V\left(R^{n}\right)=V_{n}$ and $\phi_{n}$ is the image of the standard embedding $R^{n} \rightarrow R^{n+1}$. 
Proposition 3.6. Let $\left\{V_{n}\right\}_{n \in \mathbb{N}}$ be a sequence of $\operatorname{Sp}_{2 n} R$-representations and let $\phi_{n}: V_{n} \rightarrow V_{n+1}$ be $\mathrm{Sp}_{2 n} R$-equivariant maps. Then $\mathrm{Sp}_{2 n-2 m} R$ acts trivially on the image of $V_{m}$ in $V_{n}$ if and only if there is a $\mathrm{VIC}_{R}$-module $V$ with $V\left(R^{2 n}\right)=V_{n}$ and $\phi_{n}$ is the image of the standard embedding $R^{2 n} \rightarrow R^{2 n+2}$.

In what follows we often want to treat $\mathrm{VIC}_{\mathbb{Q}}$ and $\mathrm{SI}_{\mathbb{Q}}$ uniformly. To that end we will write $\mathcal{C}$ instead of $\mathrm{VIC}_{\mathbb{Q}}$ and $\mathrm{SI}_{\mathbb{Q}}$ when we want to make a statement that is true for both categories. We will also write $G_{n}$ for $\mathrm{GL}_{n} \mathbb{Q}$ or $\mathrm{Sp}_{2 n} \mathbb{Q}$ depending on the setting.

3.3. Stability degree. Analogous to the approach by Church-Ellenberg-Farb [CEF15, Sec 3.2] we want to introduce the stability degree of $\mathcal{C}$-modules. We first make the observation that there is an injection $G_{a} \times G_{n-a} \rightarrow G_{n}$ given by a block sum. Therefore we can consider the coinvariants

$$
\mathbb{Q} \underset{\mathbb{Q} G_{n-a}}{\otimes} \operatorname{Res}_{G_{a} \times G_{n-a}}^{G_{n}} V_{n}
$$

as a $\mathbb{Q} G_{a}$-module for any $\mathbb{Q} G_{n}$-module $V_{n}$. Furthermore $\phi: V_{n} \rightarrow V_{n+1}$ induces a $G_{a}$-map

$$
\mathbb{Q} \underset{\mathbb{Q} G_{n-a}}{\otimes} V_{n} \stackrel{\phi_{*}}{\longrightarrow} \mathbb{Q} \underset{\mathbb{Q} G_{n-a}}{\otimes} V_{n+1} \longrightarrow \mathbb{Q} \underset{\mathbb{Q} G_{n+1-a}}{\otimes} V_{n+1}
$$

Definition 3.7. Let $\tau_{n, a}$ be the functor

$$
\tau_{n, a} V_{n}=\mathbb{Q} \underset{\mathbb{Q} G_{n-a}}{\otimes} \operatorname{Res}_{G_{a} \times G_{n-a}}^{G_{n}} V_{n}
$$

from $\mathbb{Q} G_{n}$-modules to $\mathbb{Q} G_{a}$-modules. We say a $\mathcal{C}$-module $V$ has injectivity degree, surjectivity degree or stability degree $\leq s$ if the map

$$
\tau_{a+n, a} V_{a+n} \stackrel{\phi_{*}}{\longrightarrow} \tau_{a+n+1, a} V_{a+n+1}
$$

is injective, surjective or bijective, respectively, for all nonnegative integers a and all $n \geq s$.

Remark 3.8. Note that if

$$
\operatorname{Res}_{G_{a} \times G_{n-a}}^{G_{n}} V_{n} \cong \bigoplus W_{i} \otimes W_{i}^{\prime},
$$

with simple $\mathbb{Q} G_{a} \otimes \mathbb{Q} G_{n-a}-$ modules $W_{i} \otimes W_{i}^{\prime}$, then

$$
\tau_{n, a} V_{n} \cong \bigoplus_{W_{i}^{\prime} \text { trivial }} W_{i} .
$$

The following two propositions are analogues to [CEF15, Lem 3.2.7] and follow immediately from the previous remark and Corollaries 2.17 and 2.18, respectively.

Proposition 3.9. Let $\lambda^{+}, \lambda^{-}$be partitions with $\ell\left(\lambda^{+}\right)+\ell\left(\lambda^{-}\right) \leq n$ and let $\mu^{+}, \mu^{-}$ be partitions with $\ell\left(\mu^{+}\right)+\ell\left(\mu^{-}\right) \leq m$. Assume further $\left|\lambda^{+}\right|+\left|\lambda^{-}\right| \leq\left|\mu^{+}\right|+\left|\mu^{-}\right|$, 
then

$$
\left[\tau_{n, m} \mathrm{GL}_{n}\left(\lambda^{+}, \lambda^{-}\right), \mathrm{GL}_{m}\left(\mu^{+}, \mu^{-}\right)\right]= \begin{cases}1 & \text { if } \mu^{+}=\lambda^{+} \text {and } \mu^{-}=\lambda^{-} \\ 0 & \text { otherwise. }\end{cases}
$$

Proposition 3.10. Let $\lambda$ be a partition with $\ell(\lambda) \leq n$ and let $\mu$ be a partition with $\ell(\mu) \leq m$. Assume further $|\lambda| \leq|\mu|$, then

$$
\left[\tau_{n, m} \operatorname{Sp}_{2 n}(\lambda), \operatorname{Sp}_{2 m}(\mu)\right]= \begin{cases}1 & \text { if } \mu=\lambda \\ 0 & \text { otherwise. }\end{cases}
$$

The next proposition is the analogue of [CEF15, Prop 3.1.7]. It turns out to be much more complicated than in the case of symmetric groups studied in [CEF15]. Later we will only need finite surjectivity degree of $M(m)$, but we give both injectivity degree and surjectivity degree for completeness sake.

Proposition 3.11. $M(m)$ has injectivity degree $\leq 0$ and surjectivity degree $\leq 2 m$.

Proof. From Remark 3.2 we get that

$$
M(m)_{a+n} \cong \mathbb{Q}\left[G_{a+n} / G_{a+n-m}\right]
$$

The functor $\tau_{a+n, a}$ takes coinvariants with respect to the $G_{n}$-action from the left, so

$$
\tau_{a+n, a} M(m)_{a+n} \cong \mathbb{Q}\left[G_{a+n} / G_{a+n-m}\right]_{G_{n}} \cong \mathbb{Q}\left[G_{n} \backslash G_{a+n} / G_{a+n-m}\right] .
$$

To understand the actions better, let us specify to the general linear case and let $G_{a+n}=\mathrm{GL}_{a+n} \mathbb{Q}$ act on the $(a+n)$-dimensional vector space with the basis

$$
\mathbb{Q}^{a+n}=\mathbb{Q}\left[e_{1}, \ldots, e_{a}, e_{a+1}, \ldots, e_{a+n}\right] .
$$

Then $G_{n}=\mathrm{GL}_{n} \mathbb{Q}$ is the subgroup acting on the subspace

$$
\mathbb{Q}^{n}=\mathbb{Q}\left[e_{a+1}, \ldots, e_{a+n}\right]
$$

and fixing $e_{1}, \ldots, e_{a}$. Similarly $G_{a+n-m}=\mathrm{GL}_{a+n-m} \mathbb{Q}$ is the subgroup acting on

$$
\mathbb{Q}^{a+n-m}=\mathbb{Q}\left[e_{m+1}, \ldots, e_{a+n}\right]
$$

and fixing $e_{1}, \ldots, e_{m}$.

The map $\phi_{*}: \tau_{a+n, a} M(m)_{a+n} \rightarrow \tau_{a+n+1, a} M(m)_{a+n+1}$ from Definition 3.7 is then given by

$$
\mathbb{Q}\left[G_{n} \backslash G_{a+n} / G_{a+n-m}\right] \rightarrow \mathbb{Q}\left[G_{n+1} \backslash G_{a+n+1} / G_{a+n+1-m}\right]
$$

which is in fact induced by the natural map

$$
G_{n} \backslash G_{a+n} / G_{a+n-m} \longrightarrow G_{n+1} \backslash G_{a+n+1} / G_{a+n+1-m}
$$

on the basis. Here we think of $G_{a+n}=\mathrm{GL}_{a+n} \mathbb{Q}$ as a subgroup of $G_{a+n+1}=$ $\mathrm{GL}_{a+n+1}$ by the standard inclusion

$$
\mathbb{Q}\left[e_{1}, \ldots, e_{a}, e_{a+1}, \ldots, e_{a+n}\right] \subset \mathbb{Q}\left[e_{1}, \ldots, e_{a}, e_{a+1}, \ldots, e_{a+n}, e_{a+n+1}\right] .
$$


Hence it suffices to consider injectivity and surjectivity for the mapping between bases.

We start with injectivity. Let $g \in G_{a+n}, x \in G_{n+1}, y \in G_{a+n+1-m}$ and assume $g^{\prime}=x g y \in G_{a+n}$. We want to prove that $g$ and $g^{\prime}$ represent the same element in $G_{n} \backslash G_{a+n} / G_{a+n-m}$. To do so, we use the following block matrix form.

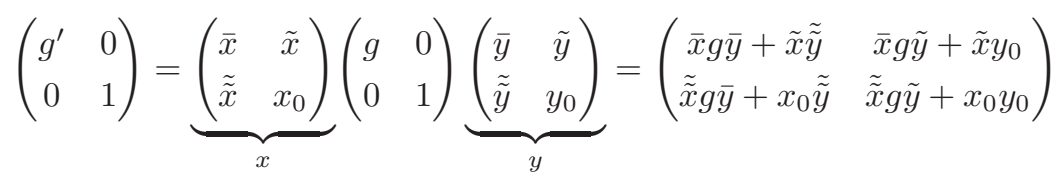

Now let us consider $\mathcal{C}=\mathrm{VIC}_{\mathbb{Q}}$. Assume first $\bar{x}$ is invertible. Then

$$
\bar{x} g \tilde{y}+\tilde{x} y_{0}=0 \Longrightarrow \tilde{y}+(\bar{x} g)^{-1} \tilde{x} y_{0}=0 \Longrightarrow-y_{0}^{-1} \tilde{y}=(\bar{x} g)^{-1} \tilde{x} .
$$

The second implication is because not both $y_{0}$ and $\tilde{y}$ can be zero as $y$ is invertible. Thus

$$
\bar{x} \cdot g \cdot\left(\bar{y}-y_{0}^{-1} \tilde{y} \tilde{\tilde{y}}\right)=\bar{x} g \bar{y}+(\bar{x} g)(\bar{x} g)^{-1} \tilde{x} \tilde{\tilde{y}}=g^{\prime},
$$

where $\bar{x} \in \mathrm{GL}_{n} \mathbb{Q}$ and $\left(\bar{y}-y_{0}^{-1} \tilde{y} \tilde{\tilde{y}}\right) \in \mathrm{GL}_{a+n-m} \mathbb{Q}$. The same argument works, when $\bar{y}$ is invertible. So assume that both $\bar{x}$ and $\bar{y}$ are not invertible. Then

$$
\tilde{x} \notin \operatorname{im} \bar{x} \quad \text { and } \quad \tilde{\tilde{y}}^{T} \notin \operatorname{im} \bar{y}^{T} .
$$

Then

$$
\bar{x}(g \tilde{y})=-y_{0} \tilde{x}
$$

implies $y_{0}=0$ and $\bar{x} g \tilde{y}=0$ and

$$
(\tilde{\tilde{x}} g) \bar{y}=-x_{0} \tilde{\tilde{y}}
$$

implies $x_{0}=0$ and $\tilde{\tilde{x}} g \bar{y}=0$. Also

$$
1=\tilde{\tilde{x}} g \tilde{y}+x_{0} y_{0}=\tilde{\tilde{x}} g \tilde{y} .
$$

Thus

$$
(\bar{x}+\tilde{x} \tilde{\tilde{x}}) \cdot g \cdot(\bar{y}+\tilde{y} \tilde{\tilde{y}})=\bar{x} g \bar{y}+\bar{x} g \tilde{y} \tilde{\tilde{y}}+\tilde{x} \tilde{\tilde{x}} g \bar{y}+\tilde{x} \tilde{\tilde{x}} g \tilde{y} \tilde{\tilde{y}}=g^{\prime} .
$$

Now consider $\mathcal{C}=\mathrm{SI}_{\mathbb{Q}}$. Denote by

$$
\Omega_{n}=\left(\begin{array}{ccccc}
0 & 1 & & & \\
-1 & 0 & & & \\
& & \ddots & & \\
& & & 0 & 1 \\
& & & -1 & 0
\end{array}\right)
$$

the Gram matrix of the standard symplectic form on a $2 n$-dimensional vector space.

Then

$$
x \cdot\left(\begin{array}{l}
g \tilde{y} \\
y_{0}
\end{array}\right)=\left(\begin{array}{l}
0 \\
1
\end{array}\right)
$$


implies that

$$
\left(\begin{array}{l}
g \tilde{y} \\
y_{0}
\end{array}\right)=\Omega_{n+1} x^{T} \Omega_{n+1}^{-1}\left(\begin{array}{l}
0 \\
1
\end{array}\right)=\Omega_{n+1}\left(\begin{array}{ll}
\tilde{x} & x_{0}
\end{array}\right)^{T} \Omega_{1}^{-1} .
$$

Thus

$$
y_{0}=\Omega_{1} x_{0}^{T} \Omega_{1}^{-1}, \quad g \tilde{y}=\Omega_{n} \tilde{\tilde{x}}^{T} \Omega_{1}^{-1} \quad \text { and } \quad \tilde{\tilde{x}} g=\Omega_{1} \tilde{y}^{T} \Omega_{n}^{-1} .
$$

Because $x$ and $y$ are symplectic we derive

$$
\tilde{y}^{T} \Omega_{n} \bar{y}=-y_{0}^{T} \Omega_{1} \tilde{\tilde{y}}, \quad \bar{x} \Omega_{n} \tilde{\tilde{x}}^{T}=-\tilde{x} \Omega_{1} x_{0}^{T} \quad \text { and } \quad \tilde{\tilde{x}} \Omega_{n} \tilde{\tilde{x}}^{T}=\Omega_{1}-x_{0} \Omega_{1} x_{0}^{T} .
$$

One can check that we can find an $\alpha \in \mathrm{Sp}_{2} \mathbb{Q}=\mathrm{SL}_{2} \mathbb{Q}$ such that $\alpha-x_{0}$ is invertible.

This is equivalent to $\alpha^{-1}-y_{0}$ being invertible. Let $\beta, \gamma \in \mathrm{GL}_{2 n} \mathbb{Q}$ such that

$$
\beta \tilde{x}=\tilde{x}\left(\alpha-x_{0}\right)^{-1} \quad \text { and } \quad \tilde{\tilde{y}} \gamma=\left(\alpha^{-1}-y_{0}\right)^{-1} \tilde{\tilde{y}}
$$

Then

$$
\tilde{x}+\beta \tilde{x} x_{0}=\beta \tilde{x} \alpha \quad \text { and } \quad \tilde{\tilde{y}}+y_{0} \tilde{\tilde{y}} \gamma=\alpha^{-1} \tilde{\tilde{y}} \gamma
$$

We can now calculate:

$$
\begin{aligned}
(\bar{x}+\beta \tilde{x} \tilde{\tilde{x}}) g(\bar{y}+\tilde{y} \tilde{\tilde{y}} \gamma) & =\bar{x} g \bar{y}+\beta \tilde{x} \tilde{\tilde{x}} g \bar{y}+\bar{x} g \tilde{y} \tilde{\tilde{y}} \gamma+\beta \tilde{x} \tilde{\tilde{x}} g \tilde{y} \tilde{\tilde{y}} \gamma \\
& =\bar{x} g \bar{y}+\beta \tilde{x} \Omega_{1}^{-1} \tilde{y}^{T} \Omega_{n} \bar{y}+\bar{x} \Omega_{n} \tilde{\tilde{x}}{ }^{T} \Omega_{1}^{-1} \tilde{\tilde{y}} \gamma+\beta \tilde{x} \tilde{\tilde{x}} \Omega_{n} \tilde{\tilde{x}}^{T} \Omega_{1}^{-1} \tilde{\tilde{y}} \gamma \\
& =\bar{x} g \bar{y}-\beta \tilde{x} \Omega_{1}^{-1} y_{0}^{T} \Omega_{1} \tilde{\tilde{y}}-\tilde{x} \Omega_{1} x_{0}^{T} \Omega_{1}^{-1} \tilde{\tilde{y}} \gamma+\beta \tilde{x}\left(\Omega_{1}-x_{0} \Omega_{1} x_{0}^{T}\right) \Omega_{1}^{-1} \tilde{\tilde{y}} \gamma \\
& =\bar{x} g \bar{y}-\beta \tilde{x} x_{0} \tilde{\tilde{y}}-\tilde{x} y_{0} \tilde{\tilde{y}} \gamma+\beta \tilde{x}\left(1-x_{0} y_{0}\right) \tilde{\tilde{y}} \gamma \\
& =\bar{x} g \bar{y}+\tilde{x} \tilde{\tilde{y}}+\beta \tilde{x} \tilde{\tilde{y}} \gamma-\left(\tilde{x}+\beta \tilde{x} x_{0}\right)\left(\tilde{\tilde{y}}+y_{0} \tilde{\tilde{y}} \beta\right) \\
& =\bar{x} g \bar{y}+\tilde{x} \tilde{\tilde{y}}=g^{\prime}
\end{aligned}
$$

Also:

$$
\begin{aligned}
(\bar{x}+\beta \tilde{x} \tilde{x}) \Omega_{n}(\bar{x}+\beta \tilde{x} \tilde{\tilde{x}})^{T} & =\bar{x} \Omega_{n} \bar{x}^{T}+\bar{x} \Omega_{n} \tilde{\tilde{x}}^{T} \tilde{x}^{T} \beta^{T}+\beta \tilde{x} \tilde{\tilde{x}} \Omega_{n} \bar{x}^{T}+\beta \tilde{x} \tilde{\tilde{x}} \Omega_{n} \tilde{\tilde{x}}^{T} \tilde{x}^{T} \beta^{T} \\
& =\bar{x} \Omega_{n} \bar{x}^{T}-\tilde{x} \Omega_{1} x_{0}^{T} \tilde{x}^{T} \beta^{T}-\beta \tilde{x} x_{0} \Omega_{1} \bar{x}^{T}+\beta \tilde{x}\left(\Omega_{1}-x_{0} \Omega_{1} x_{0}^{T}\right) \tilde{x}^{T} \beta^{T} \\
& =\bar{x} \Omega_{n} \bar{x}^{T}+\tilde{x} \Omega_{1} \tilde{x}^{T}+\beta \tilde{x} \Omega_{1} \tilde{x}^{T} \beta^{T}-\left(\tilde{x}+\beta \tilde{x} x_{0}\right) \Omega_{1}\left(\tilde{x}+\beta \tilde{x} x_{0}\right)^{T} \\
& =\bar{x} \Omega_{n} \bar{x}^{T}+\tilde{x} \Omega_{1} \tilde{x}^{T}+\beta \tilde{x} \Omega_{1} \tilde{x}^{T} \beta^{T}-\beta \tilde{x} \alpha \Omega_{1}(\beta \tilde{x} \alpha)^{T} \\
& =\bar{x} \Omega_{n} \bar{x}^{T}+\tilde{x} \Omega_{1} \tilde{x}^{T}=\Omega_{n}
\end{aligned}
$$

And analogously:

$$
(\bar{y}+\tilde{y} \tilde{\tilde{y}} \gamma)^{T} \Omega_{n}(\bar{y}+\tilde{y} \tilde{\tilde{y}} \gamma)=\Omega_{n}
$$

This proves injectivity degree $\leq 0$.

For surjectivity consider first $\mathcal{C}=\mathrm{Sl}_{\mathbb{Q}}$. Let $g \in \operatorname{Sp}_{2(a+n+1)} \mathbb{Q}$. Because $2(n+1)+$ $2(a+n+1-m)=2(a+n+1)+2(n+1-m)$, the intersection $\mathbb{Q}^{2(n+1)} \cap g \mathbb{Q}^{2(a+n+1-m)}$ is at least $2(n+1-m)$-dimensional. Assume $n \geq 2 m$, then $2(n+1-m) \geq n+2$. Therefore it cannot be an isotropic subspace of $\mathbb{Q}^{2(n+1)}$. In particular, there are vectors $v, v^{\prime} \in \mathbb{Q}^{2(n+1)} \cap g \mathbb{Q}^{2(a+n+1-m)}$ such that $\left\langle v, v^{\prime}\right\rangle=1$. We hence may find an $h_{1} \in \operatorname{Sp}_{2(a+n+1-m)} \mathbb{Q}$ that sends $\left(e_{a+n+1}, e_{a+n+1}^{\prime}\right)$ to $\left(g^{-1} v, g^{-1} v^{\prime}\right)$ and an $h_{2} \in \mathrm{Sp}_{2(n+1)} \mathbb{Q}$ that sends $\left(v, v^{\prime}\right)$ to $\left(e_{a+n+1}, e_{a+n+1}^{\prime}\right)$. Then $h_{2} g h_{1} \in \operatorname{Sp}_{2(a+n)} \mathbb{Q}$, 
thus $\mathrm{Sp}_{2(n+1)} \mathbb{Q} g \mathrm{Sp}_{2(a+n+1-m)} \mathbb{Q}$ is the image of $\mathrm{Sp}_{2 n} \mathbb{Q} h_{2} g h_{1} \mathrm{Sp}_{2(a+n-m)} \mathbb{Q}$ and surjectivity degree is $\leq 2 m$.

And finally for $\mathcal{C}=\mathrm{VIC}_{\mathbb{Q}}$ let $g \in \mathrm{GL}_{a+n+1} \mathbb{Q}$ and $n \geq 2 m$. We need to find a $g^{\prime} \in \mathrm{GL}_{a+n}$ such that

$$
\mathrm{GL}_{n+1} \mathbb{Q} \cdot g \cdot \mathrm{GL}_{a+n+1-m} \mathbb{Q}=\mathrm{GL}_{n+1} \mathbb{Q} \cdot g^{\prime} \cdot \mathrm{GL}_{a+n+1-m} \mathbb{Q} .
$$

That means we may do matrix transformations on $g$ from the left by $\mathrm{GL}_{n+1} \mathbb{Q}$ and from the right by $\mathrm{GL}_{a+n+1-m} \mathbb{Q}$ to transform to $g^{\prime}$.

Because $n \geq 2 m$, we have $(n+1)+(a+n+1-m) \geq(a+n+1)+m+1$. Thus the intersection $g^{-1} \mathbb{Q}^{n+1} \cap \mathbb{Q}^{a+n+1-m}$ is at least $(m+1)$-dimensional. Let $V$ be an $(m+1)$-dimensional subspace of this intersection. We can $g$ transform such that

$$
V=\langle\underbrace{e_{a+n+1-m}, \ldots, e_{a+n+1}}_{m+1}\rangle,
$$

$g$ is the identity on $V$ and sends $\left\langle e_{m+1}, \ldots, e_{a+n-m}\right\rangle$ to $\left\langle e_{1}, \ldots, e_{a+n-m}\right\rangle$. Because

$$
\left(g\left\langle e_{1}, \ldots, e_{m}\right\rangle\right)^{\perp} \cap V
$$

is not trivial, we may transform $g$ that it sends $\left\langle e_{1}, \ldots, e_{m}\right\rangle$ to $\mathbb{Q}^{a+n}$. In summary we transformed $g$ to have the form

$$
\left(\begin{array}{llll}
A & B & 0 & 0 \\
C & D & 0 & 0 \\
E & 0 & 1 & 0 \\
0 & 0 & 0 & 1
\end{array}\right)
$$

where the matrices $A, B, C, D, E$ have the dimensions $a \times m, a \times(a+n-2 m)$, $(n-m) \times m,(n-m) \times(a+n-m), m \times m$, respectively. And therefore $g \in$ $\mathrm{GL}_{a+n} \mathbb{Q}$, which proves surjectivity degree $\leq 2 m$.

Corollary 3.12. A $\mathcal{C}$-module $V$ that is generated in ranks $\leq m$ has surjectivity degree $\leq 2 m$.

Proof. Note that $\tau$ is right exact, thus the following commutative diagram yields the assertion.

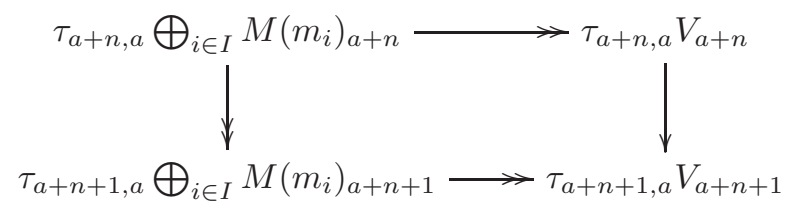

\subsection{Noetherian property.}

Definition 3.13. Let

$$
\Phi_{a} V=\bigoplus_{n \in \mathbb{N}} \tau_{a+n, a} V_{a+n}
$$

be the graded module over the graded polynomial ring $\mathbb{Q}[T] . T$ acts via

$$
\phi_{*}: \tau_{a+n, a} V_{a+n} \longrightarrow \tau_{a+n+1, a} V_{a+n+1}
$$


from Definition 3.7.

Proof of Theorems $C$ and $D$. Let $V$ be a rational $\mathrm{SI}_{\mathbb{Q}}-$ module that is finitely generated in ranks $\leq a$. Then the $\mathbb{Q}[T]$-module

$$
\Phi_{a} V=\bigoplus_{n \in \mathbb{N}} \tau_{a+n, a} V_{a+n}
$$

is finitely generated in degrees $\leq 2 a$. The submodule

$$
\Phi_{a} W \subset \Phi_{a} V
$$

is a finitely generated $\mathbb{Q}[T]$-module because $\mathbb{Q}[T]$ is a noetherian ring. Let $x_{1} \in$ $\Phi_{a} W_{n_{1}}, \ldots, x_{r} \in \Phi_{a} W_{n_{r}}$ be homogeneous generators and $w_{1} \in W_{a+n_{1}}, \ldots, w_{r} \in$ $W_{a+n_{r}}$ their respective preimages. Denote the submodule generated by $w_{1}, \ldots, w_{r}$ by $\widetilde{W} \subset W$. Then

$$
\Phi_{a} W / \widetilde{W}=0 .
$$

Let $n \geq a$. We want to conclude that $(W / \widetilde{W})_{n}=0$ to prove the assertion. Assume otherwise that $\operatorname{Sp}_{2 n}(\lambda)$ is an irreducible constituent of $(W / \widetilde{W})_{n}$. Then

$$
\tau_{n, a} \operatorname{Sp}_{2 n}(\lambda)=0
$$

which implies that

$$
\left[\operatorname{Res}_{\operatorname{Sp}_{2 a}}^{\operatorname{Sp}_{2 n} \mathbb{Q} \times \operatorname{Sp}_{2 n-2 a} \mathbb{Q}} \operatorname{Sp}_{2 n}(\lambda), \operatorname{Sp}_{2 a}(\mu) \otimes \operatorname{Sp}_{2 n-2 a}(\emptyset)\right]=0
$$

for all $\mu$. On the other hand, we know that the image of $V_{a}$ in $V_{n}$ generates $V_{n}$ as an $\mathrm{Sp}_{2 n} \mathbb{Q}$-representation. Because $\mathrm{Sp}_{2 n-2 a} \mathbb{Q}$ acts trivially on that image, the statement is equivalent to

$$
\operatorname{Ind}_{\mathrm{Sp}_{2 a} \mathbb{Q} \times \operatorname{Sp}_{2 n-2 a} \mathbb{Q}}^{\operatorname{Sp}_{2 n}} V_{a} \otimes \operatorname{Sp}_{2 n-2 a}(\emptyset) \longrightarrow V_{n}
$$

being surjective. But as seen above

$$
\left[\operatorname{Ind}_{\mathrm{Sp}_{2 a} \mathbb{Q} \times \operatorname{Sp}_{2 n-2 a} \mathbb{Q}} V_{a} \otimes \operatorname{Sp}_{2 n-2 a}(\emptyset), \operatorname{Sp}_{2 n}(\lambda)\right]=0 .
$$

Contradiction to $\operatorname{Sp}_{2 n}(\lambda)$ being a constituent of $V_{n}$.

The argument goes through exactly the same for a $\mathrm{VIC}_{\mathbb{Q}^{-}}$module $V$ that is finitely generated in ranks $\leq a$.

3.5. Representation stability. We will prove the main technical result of this paper. The idea for the proof stems from the proof of [CEF15, Prop 3.3.3].

Lemma 3.14. Let $V$ be a rational $\mathcal{C}-$ module and $s \in \mathbb{N}$. Then there is a submodule $W$ such that $W_{n}$ contains all irreducible constituents of $V_{n}$ that are indexed by (pairs of) partitions of size at least $s$.

Proof. By Corollaries 2.17 and 2.18 irreducible constituents of $V_{m}$ that are indexed by partitions of size at least $s$ only map to irreducible constituents of $V_{n}$ that are indexed by partitions with size at least $s$. Therefore the described $W$ is a submodule of $V$. 
Theorem 3.15. Let $V$ be a rational $\mathrm{VIC}_{\mathbb{Q}}-$ module or $\mathrm{SI}_{\mathbb{Q}}-$ module that is generated in finite rank, then $V$ is multiplicity stable.

Proof. Let $V$ be an $\mathrm{SI}_{\mathbb{Q}}-$ module that is generated in finite rank. Let us write

$$
V_{n} \cong \bigoplus \operatorname{Sp}_{2 n}(\lambda)^{\oplus c_{\lambda, n}}
$$

We want to prove that $c_{\lambda, n}$ is independent of large $n$.

Fix a partition $\mu$ with length $m=\ell(\mu)$ and let $W$ be the submodule of all constituents of $V$ with size at least $|\mu|+1$ as described in Lemma 3.14. Then

$$
(V / W)_{n}=V_{n} / W_{n} \cong \bigoplus_{|\lambda| \leq|\mu|} \operatorname{Sp}_{2 n}(\lambda)^{\oplus c_{\lambda, n}}
$$

Now we want to count the multiplicity of the constituent $\operatorname{Sp}_{2 m}(\mu)$ in

$$
\tau_{n, m}(V / W)_{n} \cong \bigoplus_{|\lambda| \leq|\mu|} \tau_{n, m} \operatorname{Sp}_{2 n}(\lambda)^{\oplus c_{\lambda, n}} .
$$

By Proposition 3.10, we get the equation:

$$
\left[\tau_{n, m}(V / W)_{n}, \operatorname{Sp}_{2 m}(\mu)\right]=c_{\mu, n}
$$

Because $V$ is generated in finite rank, so is $V / W$, which therefore has finite surjectivity degree. Thus

$$
c_{\mu, n}=\left[\tau_{n, m}(V / W)_{n}, \operatorname{Sp}_{2 m}(\mu)\right]
$$

is a sequence of decreasing cardinal numbers once $n$ is large enough. Because the cardinal numbers are well ordered (see for example Hönig [Hön54]), this sequence stabilizes.

By the exact same argument we prove that if $V$ is a $\mathrm{VIC}_{\mathbb{Q}}-$ module that is generated in finite rank, $\left[V_{n}, \mathrm{GL}_{n}\left(\mu^{+}, \mu^{-}\right)\right]$is independent of large $n$.

Lemma 3.16. Let $V$ be a rational $\mathcal{C}-$ module that is generated in ranks $\leq m$, then for an irreducible constituent

$$
\mathrm{GL}_{n}\left(\lambda^{+}, \lambda^{-}\right) \subset V_{n}
$$

or

$$
\operatorname{Sp}_{n}(\lambda) \subset V_{n}
$$

the length is bounded

$$
\ell\left(\lambda^{+}\right)+\ell\left(\lambda^{-}\right) \leq 2 m
$$

or

$$
\ell(\lambda) \leq 2 m
$$

respectively.

Proof. We will prove this lemma in the symplectic case using Corollary 2.6. The proof for the general linear groups goes analogously using Corollary 2.5.

The image of $V_{m}$ generates $V_{n}$ as an $\operatorname{Sp}_{2 n} \mathbb{Q}$-representation, and $\operatorname{Sp}_{2 n-2 m} \mathbb{Q}$ acts trivial on it. If $\operatorname{Sp}_{2 n}(\lambda)$ is a constituent of $V_{n}$, there must therefore be a constituent 
$\mathrm{Sp}_{2 m}(\mu)$ of $V_{m}$ such that

$$
\operatorname{Sp}_{2 m}(\mu) \otimes \operatorname{Sp}_{2 n-2 m}(\emptyset) \subset \operatorname{Res}_{\operatorname{Sp}_{2 m}}^{\operatorname{Sp}_{2 n} \mathbb{Q} \times \operatorname{Sp}_{2 n-2 m} \mathbb{Q}} \operatorname{Sp}_{2 n}(\lambda)
$$

Thus

$$
\left[\operatorname{Res}_{\mathrm{Sp}_{2 n-2 m}}^{\mathrm{Sp}_{2 n} \mathbb{Q}} \operatorname{Sp}_{2 n}(\lambda), \operatorname{Sp}_{2 n-2 m}(\emptyset)\right] \neq 0
$$

which by Corollary 2.6 implies that

$$
0=\ell(\emptyset) \geq \ell(\lambda)-2 m
$$

Proof of Theorems $A$ and $B$. Let $V$ be finitely generated in ranks $\leq m$. By Theorem 3.15, $V$ is multiplicity stable. Let $\operatorname{ker} \phi$ be the submodule of $V$ given by

$$
\operatorname{ker} \phi_{n} \subset V_{n} \text {. }
$$

Then by Theorem D, ker $\phi$ is also finitely generated, which implies that $\operatorname{ker} \phi_{n}=0$ for all $n \in \mathbb{N}$ large enough. This is injectivity.

Surjectivity is equivalent to being generated in finite rank.

Let us finally specialize to the symplectic groups. The proof for the general linear groups is the same. We want to prove that there are only finitely many partitions $\lambda$ such that $\operatorname{Sp}_{2 n}(\lambda)$ is a constituent of $V_{n}$ for some $n \in \mathbb{N}$. From Lemma 3.16, we already know that $\ell(\lambda)$ must be at most $2 m$. For every fixed $n \in \mathbb{N}$, there are certainly only finitely many partitions $\lambda$ such that $\operatorname{Sp}_{2 n}(\lambda)$ is a constituent of $V_{n}$, because the submodule $W \subset V$ defined by

$$
W_{m}= \begin{cases}0 & m<n \\ V_{m} & m \geq n\end{cases}
$$

would not be finitely generated otherwise. We now consider

$$
\tau_{n, 2 m} V_{n}
$$

for $n \geq 2 m$. For all constituents $\operatorname{Sp}_{2 n}(\lambda)$ of $V_{n}$, we know from Proposition 3.10 that

$$
\operatorname{Sp}_{4 m}(\lambda) \subset \tau_{n, 2 m} V_{n}
$$

Because $V$ has surjectivity degree $\leq 2 m$, we also know that all constituents of $V_{n}$ for $n \geq 4 m$ must already be included in the finitely generated

$$
\tau_{4 m, 2 m} V_{4 m}
$$

This finishes the proof that $V$ is uniformly representation stable.

\section{N-SERIES AND THEIR ASSOCIATED LIE ALGEBRAS}

The following definitions follow Lazard [Laz54]. He defines a generalization of a central series, such that as for the lower central series, we get a graded Lie algebra structure on the associated graded of the filtration. 
Definition 4.1. For a group $\Gamma$ a filtration $\nu \Gamma$

$$
\cdots \leq \nu_{2} \Gamma \leq \nu_{1} \Gamma=\Gamma
$$

is called an $\mathrm{N}-$ series if $\left[\nu_{i} \Gamma, \nu_{j} \Gamma\right] \leq \nu_{i+j} \Gamma$.

Definition 4.2 (Lazard [Laz54, Thm I.2.1]). The rationalized graded Lie algebra

$$
\operatorname{gr}(\nu \Gamma)=\bigoplus_{i \geq 1} \operatorname{gr}_{i}(\nu \Gamma)
$$

associated to an $N$-series $\nu$ is defined by

$$
\operatorname{gr}_{i}(\nu \Gamma)=\nu_{i} \Gamma / \nu_{i+1} \Gamma \underset{\mathbb{Z}}{\otimes} \mathbb{Q}
$$

The bracket is given by the (group) commutator.

Remark 4.3. Let $\nu$ be an $N$-series of a group $\Gamma$. Then $\Gamma$ acts via conjugation on $\nu_{i} \Gamma$ for every $i \in \mathbb{N}$ because

$$
[g, n] \in \nu_{i+1} \Gamma \leq \nu_{i} \Gamma
$$

for $g \in \Gamma$ and $n \in \nu_{i} \Gamma$. The same argument shows that $\Gamma$ acts trivially on $\operatorname{gr}_{i}(\nu \Gamma)$.

Definition 4.4. For every group $\Gamma$ its lower central series $\gamma \Gamma$ defined by

$$
\gamma_{1} \Gamma=\Gamma \quad \text { and } \quad \gamma_{i+1}=\left[\Gamma, \gamma_{i} \Gamma\right]
$$

is an $N$-series. $\operatorname{gr}(\gamma \Gamma)$ is sometimes called the Malcev Lie algebra associated to $\Gamma$.

Example 4.5. Let $\Gamma=F_{n}$ be the free group with $n$ generators. Then its Malcev Lie algebra is the free Lie algebra $\mathcal{L}_{n}$ with $n$ generators.

Definition 4.6. For the automorphism group $\operatorname{Aut}(\Gamma)$ of a group $\Gamma$,

$$
\alpha_{i} \operatorname{Aut}(\Gamma)=\operatorname{ker}\left(\operatorname{Aut}(\Gamma) \rightarrow \operatorname{Aut}\left(\Gamma / \gamma_{i+1} \Gamma\right)\right)
$$

is called the Andreadakis filtration.

Andreadakis [And65, Thm 1.1(ii)] showed that $\alpha$ is an $N$-series of $\alpha_{1} \operatorname{Aut}(\Gamma)$.

5. Torelli subgroups of The AUtomorphism Groups of FreE Groups

Let $F_{n}$ denote the free group on $n$ generators, then its abelianization is

$$
\mathbb{Z}^{n} \cong F_{n} /\left[F_{n}, F_{n}\right]
$$

The quotient map induces a group homomorphism

$$
\operatorname{Aut}\left(F_{n}\right) \longrightarrow \operatorname{Aut}\left(\mathbb{Z}^{n}\right)=\mathrm{GL}_{n}(\mathbb{Z})
$$

on their automorphism groups because the commutator subgroup $\left[F_{n}, F_{n}\right] \leq F_{n}$ is characteristic. Nielsen [Nie18] proved that $\operatorname{Aut}\left(F_{n}\right)$ is generated by the permutations of the generators $x_{1}, \ldots, x_{n}$ and the following two automorphisms.

$$
x_{i} \mapsto\left\{\begin{array} { l l } 
{ x _ { 1 } ^ { - 1 } } & { i = 1 } \\
{ x _ { i } } & { i \neq 1 }
\end{array} \text { and } \quad x _ { i } \mapsto \left\{\begin{array}{ll}
x_{1} x_{2} & i=1 \\
x_{i} & i \neq 1
\end{array}\right.\right.
$$


The images of these automorphisms also generate $\mathrm{GL}_{n} \mathbb{Z}$. Hence the homomorphism between the automorphism groups is surjective.

The Torelli subgroup $\mathrm{IA}_{n}$ is defined as the kernel, so we get the following short exact sequence.

$$
1 \rightarrow \mathrm{IA}_{n} \rightarrow \operatorname{Aut}\left(F_{n}\right) \rightarrow \mathrm{GL}_{n} \mathbb{Z} \rightarrow 1
$$

As for every short exact sequence, we get an outer action of $\mathrm{GL}_{n} \mathbb{Z}$ on $\mathrm{IA}_{n}$, ie a group homomorphism

$$
\mathrm{GL}_{n} \mathbb{Z} \longrightarrow \operatorname{Out}\left(\mathrm{IA}_{n}\right)=\operatorname{Aut}\left(\mathrm{IA}_{n}\right) / \operatorname{Inn}\left(\mathrm{IA}_{n}\right) .
$$

This homomorphism is given as follows. Let $g \in \mathrm{GL}_{n} \mathbb{Z}$ and $\tilde{g} \in \operatorname{Aut}\left(F_{n}\right)$ a preimage of $g$. Then conjugation by $\tilde{g}$ is an automorphism of $\mathrm{IA}_{n}$. Another preimage of $g$ is $\tilde{g} h$ for some $h \in \mathrm{IA}_{n}$. Then conjugation by $\tilde{g} h$ is conjugation by $\tilde{g}$ composed with the inner automorphism defined by $h$.

This outer action gives rise to a $\mathrm{GL}_{n} \mathbb{Z}$-representation on the abelianization $H_{1}\left(\mathrm{IA}_{n} ; \mathbb{Z}\right)$ of $\mathrm{IA}_{n}$ because inner automorphisms act trivially. After rationalizing the $\mathrm{GL}_{n} \mathbb{Z}$-representation

$$
H_{1}\left(\mathrm{IA}_{n} ; \mathbb{Q}\right) \cong \bigwedge^{2} \mathbb{Q}^{n} \otimes\left(\mathbb{Q}^{n}\right)^{*}
$$

was computed for example by Kawazumi [Kaw06, Thm 6.1]. It is clearly a restriction of a $\mathrm{GL}_{n} \mathbb{Q}$-representation.

Even more, for every morphism

$$
(f, C) \in \operatorname{Hom}_{\mathrm{VIC}}\left(\mathbb{Q}^{m}, \mathbb{Q}^{n}\right)
$$

we get a unique section $s: \mathbb{Q}^{n} \rightarrow \mathbb{Q}^{m}$ of $f$ such that $C=\operatorname{ker} s$ and therefore a well-defined map

$$
H_{1}\left(\mathrm{IA}_{m} ; \mathbb{Q}\right) \cong \bigwedge^{2} \mathbb{Q}^{m} \otimes\left(\mathbb{Q}^{m}\right)^{*} \longrightarrow H_{1}\left(\mathrm{IA}_{n} ; \mathbb{Q}\right) \cong \bigwedge^{2} \mathbb{Q}^{n} \otimes\left(\mathbb{Q}^{n}\right)^{*},
$$

which turns $\left\{H_{1}\left(\mathrm{IA}_{n} ; \mathbb{Q}\right)\right\}_{n \in \mathbb{N}}$ into a $\mathrm{VIC}_{\mathbb{Q}^{-}}$module.

For a morphism

$$
(f, C) \in \operatorname{Hom}_{\mathrm{VIC}_{\mathbb{Z}}}\left(\mathbb{Z}^{m}, \mathbb{Z}^{n}\right) \cong \mathrm{GL}_{n} \mathbb{Z} / \mathrm{GL}_{n-m} \mathbb{Z}
$$

this can be traced to a group homomorphism

$$
\mathrm{IA}_{m} \rightarrow \mathrm{IA}_{n}
$$

up to inner automorphism of $\mathrm{IA}_{n}$. Here is the reason. Let $K \subset \operatorname{Aut}\left(F_{n}\right)$ be the preimage of $\mathrm{GL}_{n-m} \mathbb{Z}$ of the composition

$$
\operatorname{Aut}\left(F_{n}\right) \longrightarrow \mathrm{GL}_{n} \mathbb{Z} \longrightarrow \operatorname{Hom}_{\mathrm{VIC}_{\mathbb{Z}}}\left(\mathbb{Z}^{m}, \mathbb{Z}^{n}\right) \cong \mathrm{GL}_{n} \mathbb{Z} / \mathrm{GL}_{n-m} \mathbb{Z} .
$$

For $(f, C)$ we find an automorphism $g \in \operatorname{Aut}\left(F_{n}\right)$, which is uniquely determined up to right multiplication by an element of $K$. Conjugating by $g$ gives an automorphism of $\mathrm{IA}_{n}$ which can be restricted to a map $\mathrm{IA}_{m} \rightarrow \mathrm{IA}_{n}$. Because $\operatorname{Aut}\left(F_{n-m}\right) \subset K$ surjects to $\mathrm{GL}_{n-m} \mathbb{Z}$, we can find for every $k \in K$ an $h \in \mathrm{IA}_{n}$ such that $h k^{-1} \in$ $\operatorname{Aut}\left(F_{n-m}\right)$. Since $\operatorname{Aut}\left(F_{n-m}\right)$ commutes with $\mathrm{IA}_{m}$, the conjugation by $g k$ is the 
same as the conjugation by $g h$ when restricted to $\mathrm{IA}_{m}$. Thus $\mathrm{IA}_{m} \rightarrow \mathrm{IA}_{n}$ is well defined up to inner automorphism of $\mathrm{IA}_{n}$.

This group monomorphism induces a map

$$
H_{1}\left(\mathrm{IA}_{m} ; \mathbb{Z}\right) \longrightarrow H_{1}\left(\mathrm{IA}_{n} ; \mathbb{Z}\right) .
$$

This map is well defined and natural because the group monomorphism is well defined up to inner automorphisms of $\mathrm{IA}_{n}$.

As already been pointed out in [CF13, Sec 6.2]

$$
H_{1}\left(\mathrm{IA}_{n} ; \mathbb{Q}\right) \cong \bigwedge^{2} \mathbb{Q}^{n} \otimes\left(\mathbb{Q}^{n}\right)^{*} \cong \mathrm{GL}_{n}(\square, \emptyset) \oplus \mathrm{GL}_{n}(\boxminus, \square)
$$

for all $n \geq 3$ is uniformly representation stable.

5.1. Lower central series of $\mathrm{IA}_{n}$. Let us first consider the lower central series $\gamma \mathrm{IA}_{n}$ (see Definition 4.4) of the Torelli subgroups $\mathrm{IA}_{n}$.

Proposition 5.1. $\left\{\operatorname{gr}_{i}\left(\gamma \mathrm{IA}_{n}\right)\right\}_{n \in \mathbb{N}}$ gives rise to a $\mathrm{VIC}_{\mathbb{Z}}-$ module.

Proof. Because $\gamma_{i} \mathrm{IA}_{n}$ is a characteristic subgroup of $\mathrm{IA}_{n}$ which is normal in $\operatorname{Aut}\left(F_{n}\right)$, the latter acts on $\gamma_{i} \mathrm{IA}_{n}$ by conjugation. For $m \leq n$, the automorphism group $\operatorname{Aut}\left(F_{n}\right)$ gives a group homomorphism

$$
\gamma_{i} \mathrm{IA}_{m} \longrightarrow \gamma_{i} \mathrm{IA}_{n}
$$

that descends to the quotients

$$
\operatorname{gr}_{i}\left(\gamma \mathrm{IA}_{m}\right) \longrightarrow \operatorname{gr}_{i}\left(\gamma \mathrm{IA}_{n}\right)
$$

Given $g \in \operatorname{Aut}\left(F_{n}\right)$ and $h \in \operatorname{Aut}\left(F_{m}\right)$ each, the composition

$$
\operatorname{gr}_{i}\left(\gamma \mathrm{IA}_{l}\right) \stackrel{h}{\longrightarrow} \operatorname{gr}_{i}\left(\gamma \mathrm{IA}_{m}\right) \stackrel{g}{\longrightarrow} \operatorname{gr}_{i}\left(\gamma \mathrm{IA}_{n}\right)
$$

is given by $g h \in \operatorname{Aut}\left(F_{n}\right)$.

Clearly $\operatorname{Aut}\left(F_{n-m}\right) \subset \operatorname{Aut}\left(F_{n}\right)$ acts trivially on

$$
\operatorname{gr}_{i}\left(\gamma \operatorname{IA}_{m}\right) \subset \operatorname{gr}_{i}\left(\gamma \mathrm{IA}_{n}\right)
$$

because is commutes with all subquotients of $\operatorname{Aut}\left(F_{m}\right)$. Furthermore, by definition

$$
\left[\mathrm{IA}_{n}, \gamma_{i} \mathrm{IA}_{n}\right]=\gamma_{i+1} \mathrm{IA}_{n}
$$

Thus also $\operatorname{IA}_{n} \subset \operatorname{Aut}\left(F_{n}\right)$ acts trivially on

$$
\operatorname{gr}_{i}\left(\gamma \operatorname{IA}_{m}\right) \subset \operatorname{gr}_{i}\left(\gamma \mathrm{IA}_{n}\right) .
$$

Therefore

$$
\operatorname{Aut}\left(F_{n}\right) / \mathrm{IA}_{n} \cdot \operatorname{Aut}\left(F_{n-m}\right)
$$

gives rise to a homomorphism

$$
\operatorname{gr}_{i}\left(\gamma \mathrm{IA}_{m}\right) \longrightarrow \operatorname{gr}_{i}\left(\gamma \mathrm{IA}_{n}\right) .
$$


But this is isomorphic to

$$
\operatorname{Aut}\left(F_{n}\right) / \mathrm{IA}_{n} /\left(\mathrm{IA}_{n} \cdot \operatorname{Aut}\left(F_{n-m}\right)\right) / \mathrm{IA}_{n} \cong \mathrm{GL}_{n} \mathbb{Z} / \mathrm{GL}_{n-m} \mathbb{Z}
$$

because $\operatorname{IA}_{n} \cap \operatorname{Aut}\left(F_{n-m}\right)=\mathrm{IA}_{n-m}$.

This defines a functor $V: \mathrm{VIC}_{\mathbb{Z}} \rightarrow \mathbb{Q}-$ mod with

$$
V_{n}=\operatorname{gr}_{i}\left(\gamma \mathrm{IA}_{n}\right)
$$

Proposition 5.2. Let $V$ be a rational $\mathrm{VIC}_{\mathbb{Q}}$-module which is uniformly representation stable and assume $V_{n}$ is finite dimensional for every $n \in \mathbb{N}$. Then the $k$ th degrees $\mathcal{L}_{k}(V)$ of the free Lie algebra generated by $V$ is a rational $\mathrm{VIC}_{\mathbb{Q}}$-module which is uniformly representation stable and $\mathcal{L}_{k}\left(V_{n}\right)$ is finite dimensional for all $n \in \mathbb{N}$.

Proof. Clearly $\mathcal{L}_{k}$ is a functor thus $\mathcal{L}_{k}(V)$ is certainly a $\mathrm{VIC}_{\mathbb{Q}}$-module. For the other assertions we adopt the methods used in the proof of [CF13, Thm 5.3].

Because the Chevalley-Eilenberg homology of a free Lie algebra $\mathcal{L}(V)$ is given by

$$
H_{i}(\mathcal{L}(V))= \begin{cases}\mathbb{Q} & i=0 \\ \mathcal{L}_{1}(V)=V & i=1, \\ 0 & i>1,\end{cases}
$$

for every $k \geq 2$ there is an exact sequence

$0 \longrightarrow\left(\bigwedge^{k} \mathcal{L}(V)\right)_{k} \longrightarrow\left(\bigwedge^{k-1} \mathcal{L}(V)\right)_{k} \longrightarrow \cdots \longrightarrow\left(\bigwedge^{2} \mathcal{L}(V)\right)_{k} \longrightarrow \mathcal{L}_{k}(V) \longrightarrow 0$ where $\left(\bigwedge^{i} \mathcal{L}(V)\right)_{k}$ is the $k$ th degree part of $\bigwedge^{i} \mathcal{L}(V)$, which is given by all direct summands

$$
\bigwedge^{i_{1}} \mathcal{L}_{k_{1}}(V) \otimes \cdots \otimes \bigwedge^{i_{r}} \mathcal{L}_{k_{r}}(V)
$$

with $k_{1}<\cdots<k_{r}$ and $\sum i_{j} \cdot k_{j}=k$ and $\sum i_{j}=i$.

We can use Theorem 2.11 and Proposition 2.15 to deduce by induction that every term except the last of the exact sequence are sequences of finite dimensional rational $\mathrm{GL}_{n} \mathbb{Q}$-representations that are uniformly representation stable. This implies that the last term $\mathcal{L}_{k}(V)$ is also a sequence of finite dimensional rational $\mathrm{GL}_{n} \mathbb{Q}$-representations that is uniformly representation stable.

Proof of Theorem E. $\operatorname{gr}\left(\gamma \mathrm{IA}_{n}\right)$ is generated in the first degree

$$
\operatorname{gr}_{1}\left(\gamma \mathrm{IA}_{n}\right) \cong H_{1}\left(\mathrm{IA}_{n} ; \mathbb{Q}\right)
$$

Thus there is a graded epimorphism

$$
\mathcal{L}\left(H_{1}\left(\mathrm{IA}_{n} ; \mathbb{Q}\right)\right) \longrightarrow \operatorname{gr}\left(\gamma \mathrm{IA}_{n}\right)
$$

from the free Lie algebra on $H_{1}\left(\mathrm{IA}_{n} ; \mathbb{Q}\right)$.

The epimorphism

$$
\mathcal{L}_{i}\left(H_{1}\left(\mathrm{IA}_{n} ; \mathbb{Q}\right)\right) \longrightarrow \operatorname{gr}_{i}\left(\gamma \mathrm{IA}_{n}\right)
$$


is a $\mathrm{GL}_{n} \mathbb{Z}$-equivariant map because it is induced by the (group) commutator, which commutes with group homomorphisms. Then because restrictions of irreducible rational $\mathrm{GL}_{n} \mathbb{Q}$-representations to $\mathrm{GL}_{n} \mathbb{Z}$ are irreducible (see Section 2.9), the quotient $\operatorname{gr}_{i}\left(\gamma \mathrm{IA}_{n}\right)$ is also a finite dimensional rational $\mathrm{GL}_{n} \mathbb{Q}$-representation.

We were not able to construct a $\mathrm{VIC}_{\mathbb{Q}^{-}}$module structure on $\left\{\operatorname{gr}_{i}\left(\gamma \mathrm{IA}_{n}\right)\right\}_{n \in \mathbb{N}}$. We can however find a $\mathrm{VIC}_{\mathbb{Q}^{-}}$module $V$ for every $i \in \mathbb{N}$ such that $V_{n} \cong \operatorname{gr}_{i}\left(\gamma \mathrm{IA}_{n}\right)$ for all large enough $n \in \mathbb{N}$. To do so we appeal to Proposition 3.5.

Theorem 5.3. Fix $i \in \mathbb{N}$. There is a rational $\mathrm{VIC}_{\mathbb{Q}}-$ module $V$ such that $V_{n}$ and $\operatorname{gr}_{i}\left(\gamma \mathrm{IA}_{n}\right)$ are isomorphic $\mathrm{GL}_{n} \mathbb{Q}$-representations for all large enough $n \in \mathbb{N}$.

Proof. We have already observed that

$$
\mathcal{L}_{i}\left(H_{1}\left(\mathrm{IA}_{?} ; \mathbb{Q}\right)\right) \longrightarrow \operatorname{gr}_{i}\left(\gamma \mathrm{IA}_{?}\right)
$$

is an epimorphism of $\mathrm{VIC}_{\mathbb{Z}^{-}}$modules. Also $\mathcal{L}_{i}\left(H_{1}\left(\mathrm{IA}_{?} ; \mathbb{Q}\right)\right)$ is a uniformly representation stable $\mathrm{VIC}_{\mathbb{Q}^{-}}$module. Let $N^{+}$be the maximum of all values $\ell\left(\lambda^{+}\right)$such that $\mathrm{GL}_{n}\left(\lambda^{+}, \lambda^{-}\right)$(for some $\lambda^{-}$) appears as a constituent in $\mathcal{L}_{i}\left(H_{1}\left(\mathrm{IA}_{n} ; \mathbb{Q}\right)\right.$ ) for some $n \in \mathbb{N}$. Similarly let $N^{-}$be the maximum of all $\lambda^{-}$for which a $\operatorname{GL}_{n}\left(\lambda^{+}, \lambda^{-}\right)$ is a constituent in $\mathcal{L}_{i}\left(H_{1}\left(\mathrm{IA}_{n} ; \mathbb{Q}\right)\right)$ for some $n \in \mathbb{N}$. Then for all $n \geq N=$ $N^{+}+N^{-}+1$ by the analysis of Section 2.9 two nonisomorphic irreducible constituent of the $\mathrm{GL}_{n} \mathbb{Q}$-representation $\mathcal{L}_{i}\left(H_{1}\left(\mathrm{IA}_{n} ; \mathbb{Q}\right)\right)$ cannot restrict to isomorphic $\mathrm{GL}_{n} \mathbb{Z}$-representations. This means for $n \geq N$, there is a unique way to extend $\operatorname{gr}_{i}\left(\gamma \mathrm{IA}_{n}\right)$ to a rational $\mathrm{GL}_{n} \mathbb{Q}$-representation such that

$$
\mathcal{L}_{i}\left(H_{1}\left(\mathrm{IA}_{n} ; \mathbb{Q}\right)\right) \longrightarrow \operatorname{gr}_{i}\left(\gamma \mathrm{IA}_{n}\right)
$$

is $\mathrm{GL}_{n} \mathbb{Q}$-equivariant.

Let

$$
V_{n}= \begin{cases}0 & n<N \\ \operatorname{gr}_{i}\left(\gamma \mathrm{IA}_{n}\right) & n \geq N\end{cases}
$$

be a sequence of $\mathrm{GL}_{n} \mathbb{Q}$-representations and let

$$
\phi_{n}: V_{n} \longrightarrow V_{n+1}
$$

be the image of the standard embedding $\mathbb{Z}^{n} \rightarrow \mathbb{Z}^{n+1}$ if $n \geq N$ and zero otherwise. $V_{n+1}$ only has irreducible constituents $\mathrm{GL}_{n+1}\left(\lambda^{+}, \lambda^{-}\right)$with $\ell\left(\lambda^{+}\right) \leq N^{+}$and $\ell\left(\lambda^{-}\right) \leq N^{-}$and by Corollary 2.17 the restriction $\operatorname{Res}_{\mathrm{GL}_{n} \mathbb{Q}}^{\mathrm{GL}_{n+1}} V_{n+1}$ has therefore also only constituents $\operatorname{GL}_{n}\left(\mu^{+}, \mu^{-}\right)$with $\ell\left(\mu^{+}\right) \leq N^{+}$and $\ell\left(\mu^{-}\right) \leq N^{-}$. Therefore $\phi_{n}$ is $\mathrm{GL}_{n} \mathbb{Q}$-equivariant.

Using Proposition 3.5, it remains to show that $\mathrm{GL}_{n-m} \mathbb{Q}$ acts trivially on the image of $V_{m}$ in $V_{n}$. This property is transferred from the $\mathrm{VIC}_{\mathbb{Q}^{-}}$module $\mathcal{L}_{i}\left(H_{1}\left(\mathrm{IA}_{\text {? }} ; \mathbb{Q}\right)\right)$ by the following argument. We already know that the image of $\mathcal{L}_{i}\left(H_{1}\left(\mathrm{IA}_{m} ; \mathbb{Q}\right)\right)$ is inside

$$
\mathcal{L}_{i}\left(H_{1}\left(\mathrm{IA}_{n} ; \mathbb{Q}\right)\right)^{\mathrm{GL}_{n-m} \mathbb{Q}}
$$


which maps to

$$
V_{n}^{\mathrm{GL}_{n-m} \mathbb{Q}} .
$$

Because

$$
\mathcal{L}_{i}\left(H_{1}\left(\mathrm{IA}_{m} ; \mathbb{Q}\right)\right) \longrightarrow V_{m}
$$

is surjective, $V_{m}$, too, must map to $V_{n}^{\mathrm{GL}_{n-m} \mathbb{Q}}$.

Proof of Theorem $F$. Let $V$ be the $\mathrm{VIC}_{\mathbb{Q}^{-}}$-module from Theorem 5.3 such that $V_{n}=$ $\operatorname{gr}_{i}\left(\gamma \mathrm{IA}_{n}\right)$ for all large enough $n \in \mathbb{N}$. Then by its description as a $\mathrm{VIC}_{\mathbb{Z}}-$ module it restricts to the truncation of the $\mathrm{FI}$-module described in [CEF15, Ex 7.3.6].

We will use the result [CEF15, Thm 7.3.8] that $V$ is (the submodule of) a finitely generated $\mathrm{FI}$-module and thereby a finitely generated $\mathrm{VIC}_{\mathbb{Q}}$-module. (Djament [Dja16, Prop 7.2] proves that $\operatorname{gr}_{i}\left(\gamma \mathrm{IA}_{\text {? }}\right)$ is a $\mathrm{VIC}_{\mathbb{Z}}$-module generated in finite rank. $)$

Our Theorem A implies then that $V$ is uniformly representation stable.

5.2. Johnson filtration of $\mathrm{IA}_{n}$. Let us consider the Andreadakis filtration $\alpha \operatorname{Aut}\left(F_{n}\right)$ (see Definition 4.6) of the automorphism group $\operatorname{Aut}\left(F_{n}\right)$. This is an $N$-series of

$$
\alpha_{1} \operatorname{Aut}\left(F_{n}\right)=\operatorname{ker}\left(\operatorname{Aut}\left(F_{n}\right) \rightarrow \operatorname{Aut}\left(F_{n} / \gamma_{2} F_{n}\right)\right)=\mathrm{IA}_{n}
$$

and is often called the Johnson filtration $\alpha \mathrm{IA}_{n}$ of $\mathrm{IA}_{n}$ because of Johnson's work on the Torelli subgroups of the mapping class groups of surfaces that started out with [Joh80].

Proposition 5.4. $\left\{\operatorname{gr}_{i}\left(\alpha \mathrm{IA}_{n}\right)\right\}_{n \in \mathbb{N}}$ gives rise to a $\mathrm{VIC}_{\mathbb{Z}}-$ module.

Proof. We follow the same strategy as in the proof of Proposition 5.1. $\operatorname{Aut}\left(F_{n}\right)$ acts on its normal subgroup $\alpha_{i} \mathrm{IA}_{n}$ by conjugation. For $m \leq n$, this action induces group homomorphisms

$$
\alpha_{i} \mathrm{IA}_{m} \longrightarrow \alpha_{i} \mathrm{IA}_{n}
$$

and

$$
\operatorname{gr}_{i}\left(\alpha \mathrm{IA}_{m}\right) \longrightarrow \operatorname{gr}_{i}\left(\alpha \mathrm{IA}_{n}\right) .
$$

It is clear that $\operatorname{Aut}\left(F_{n-m}\right) \subset \operatorname{Aut}\left(F_{n}\right)$ acts trivially on

$$
\operatorname{gr}_{i}\left(\alpha \mathrm{IA}_{m}\right) \subset \operatorname{gr}_{i}\left(\alpha \mathrm{IA}_{n}\right) .
$$

Further

$$
\left[\mathrm{IA}_{n}, \alpha_{i} \mathrm{IA}_{n}\right] \subset \alpha_{i+1} \mathrm{IA}_{n}
$$

follows from [And65, Thm 1.1(ii)]. Thus also $\mathrm{IA}_{n} \subset \operatorname{Aut}\left(F_{n}\right)$ acts trivially on

$$
\operatorname{gr}_{i}\left(\alpha \mathrm{IA}_{m}\right) \subset \operatorname{gr}_{i}\left(\alpha \mathrm{IA}_{n}\right) .
$$

By same argument as in the proof of Proposition 5.1, this construction gives a functor $V: \mathrm{VIC}_{\mathbb{Z}} \rightarrow \mathbb{Q}$-mod with

$$
V_{n}=\operatorname{gr}_{i}\left(\alpha \mathrm{IA}_{n}\right)
$$

In the case of the Johnson filtration we do not know whether $\operatorname{gr}\left(\alpha \mathrm{IA}_{n}\right)$ is generated in degree one as a Lie algebra. (Although it was conjectured by Andreadakis 
that $\alpha_{i} \mathrm{IA}_{n}=\gamma_{i} \mathrm{IA}_{n}$ for all $i, n \in \mathbb{N}$.) Luckily we have another tool at handthe Johnson homomorphism. As explained by Satoh [Sat16, Sec 3.4] there is a $\mathrm{GL}_{n} \mathbb{Z}$-equivariant monomorphism

$$
\operatorname{gr}_{i}\left(\alpha \mathrm{IA}_{n}\right) \longleftrightarrow \operatorname{Hom}_{\mathbb{Q}}\left(H_{1}\left(F_{n} ; \mathbb{Q}\right), \operatorname{gr}_{i+1}\left(\gamma F_{n}\right)\right) \cong\left(\mathbb{Q}^{n}\right)^{*} \otimes \mathcal{L}_{i+1}\left(\mathbb{Q}^{n}\right)
$$

By the same arguments used for Theorem E, we can infer the following proposition that also has been stated in the introduction of [Sat16, Sec 4] without a proof.

Proposition 5.5. The natural $\mathrm{GL}_{n} \mathbb{Z}$-representation on $\operatorname{gr}_{i}\left(\alpha \mathrm{IA}_{n}\right)$ extends to $a$ rational $\mathrm{GL}_{n} \mathbb{Q}$-representation.

As for the lower central series we can combine Proposition 5.4 and Proposition 5.5 to get the following theorem.

Theorem 5.6. Fix $i \in \mathbb{N}$. There is a rational $\mathrm{VIC}_{\mathbb{Q}}-$ module $V$ such that $V_{n}$ and $\operatorname{gr}_{i}\left(\alpha \mathrm{IA}_{n}\right)$ are isomorphic $\mathrm{GL}_{n} \mathbb{Q}$-representations for all large enough $n \in \mathbb{N}$.

Proof of Theorem $H$. Let $V$ be the $\mathrm{VIC}_{\mathbb{Q}^{-}}$-module from Theorem 5.6 such that $V_{n}=$ $\operatorname{gr}_{k}\left(\alpha \mathrm{IA}_{n}\right)$ for all large enough $n \in \mathbb{N}$.

Church and Putman [CP15] consider the groups $\mathrm{IA}_{n}$ as an $\mathrm{FI}$-group. They apply their [CP15, Thm G] to prove their [CP15, Thm C]. In the proof of the former theorem in [CP15, Claim 2] it is stated that $W(k)$ is boundedly generated. But $W(k)_{n} \otimes_{\mathbb{Z}} \mathbb{Q}$ is $\operatorname{gr}_{k}\left(\alpha \mathrm{IA}_{n}\right)=V_{n}$ for all large enough $n \in \mathbb{N}$. Because every $V_{n}$ is finite dimensional (see [CP15, Prop 3.2]), $V$ is a finitely generated FI-module and thus certainly a finitely generated $\mathrm{VIC}_{\mathbb{Q}}-$ module. (Djament [Dja16, Prop 7.3] proves that $\operatorname{gr}_{i}\left(\alpha \mathrm{IA}_{\text {? }}\right)$ is a $\mathrm{VIC}_{\mathbb{Z}}-$ module generated in finite rank independently.)

Our Theorem A implies then that $V$ is uniformly representation stable.

\section{TORElli SUbGroups OF THE MAPPING ClASS GROUPS OF SURFACES}

Let $\Sigma_{g, 1}$ denote the compact, oriented genus $g$ surface with one boundary component. The mapping class group $\operatorname{Mod}\left(\Sigma_{g, 1}\right)$ is defined as the discrete group $\pi_{0}$ Homeo $^{+}\left(\Sigma_{g, 1}, \partial \Sigma_{g, 1}\right)$ of isotopy classes of orientation-preserving homeomorphisms of $\Sigma_{g, 1}$ that fix the boundary pointwise. The action of $\operatorname{Mod}\left(\Sigma_{g, 1}\right)$ on $H_{1}\left(\Sigma_{g, 1} ; \mathbb{Z}\right) \cong$ $\mathbb{Z}^{2 g}$ is symplectic and the Torelli subgroup $\mathcal{I}_{g, 1}$ is defined to be the kernel of this action. In fact, there is a short exact sequence

$$
1 \rightarrow \mathcal{I}_{g, 1} \rightarrow \operatorname{Mod}\left(\Sigma_{g, 1}\right) \rightarrow \operatorname{Sp}\left(H_{1}\left(\Sigma_{g, 1} ; \mathbb{Z}\right)\right) \cong \operatorname{Sp}_{2 g} \mathbb{Z} \rightarrow 1
$$

Thus we get an $\mathrm{Sp}_{2 g} \mathbb{Z}$-representation on the abelianzation $H_{1}\left(\mathcal{I}_{g, 1} ; \mathbb{Z}\right)$ of $\mathcal{I}_{g, 1}$, which after rationalizing can be seen to be a restriction of a $\operatorname{Sp}\left(H_{1}\left(\Sigma_{g, 1} ; \mathbb{Q}\right)\right) \cong$ $\mathrm{Sp}_{2 g} \mathbb{Q}$-representation

$$
H_{1}\left(\mathcal{I}_{g, 1} ; \mathbb{Q}\right) \cong \bigwedge^{3} H_{1}\left(\Sigma_{g, 1} ; \mathbb{Z}\right) \otimes \mathbb{Q}=\bigwedge^{3} H_{1}\left(\Sigma_{g, 1} ; \mathbb{Q}\right)
$$

as it has been computed by Johnson [Joh85, Thm 3(a)]. And for every isometry

$$
H_{1}\left(\Sigma_{g, 1} ; \mathbb{Q}\right) \longrightarrow H_{1}\left(\Sigma_{g^{\prime}, 1} ; \mathbb{Q}\right)
$$


there is map

$$
H_{1}\left(\mathcal{I}_{g, 1} ; \mathbb{Q}\right) \cong \bigwedge^{3} H_{1}\left(\Sigma_{g, 1} ; \mathbb{Q}\right) \longrightarrow H_{1}\left(\mathcal{I}_{g^{\prime}, 1} ; \mathbb{Q}\right) \cong \bigwedge^{3} H_{1}\left(\Sigma_{g^{\prime}, 1} ; \mathbb{Q}\right),
$$

which turns $\left\{H_{1}\left(\mathcal{I}_{g, 1} ; \mathbb{Q}\right)\right\}_{g \in \mathbb{N}}$ into an SI-module.

As it has already been pointed out by in [CF13, Sec 6.1]

$$
H_{1}\left(\mathcal{I}_{g, 1} ; \mathbb{Q}\right) \cong \bigwedge^{3} H_{1}\left(\Sigma_{g, 1} ; \mathbb{Q}\right) \cong \operatorname{Sp}_{2 g}(\boxminus) \oplus \operatorname{Sp}_{2 g}(\square)
$$

for all $g \geq 3$ is uniformly representation stable.

We will consider two N-series of $\mathcal{I}_{g, 1}$. Denote the lower central series by $\gamma \mathcal{I}_{g, 1}$. To construct the Johnson filtration of $\mathcal{I}_{g, 1}$, consider the classical inclusion

$$
\operatorname{Mod}\left(\Sigma_{g, 1}\right) \hookrightarrow \operatorname{Aut}\left(F_{2 g}\right)
$$

and define

$$
\alpha_{i} \mathcal{I}_{g, 1}=\operatorname{Mod}\left(\Sigma_{g, 1}\right) \cap \alpha_{i} \mathrm{IA}_{2 g}=\operatorname{ker}\left(\operatorname{Mod}\left(\Sigma_{g, 1}\right) \rightarrow \operatorname{Aut}\left(F_{2 g} / \gamma_{i+1} F_{2 g}\right)\right) .
$$

This construction immediately implies that $\alpha \mathcal{I}_{g, 1}$ is an N-series of $\mathcal{I}_{g, 1}$ because $\alpha \mathrm{IA}_{2 g}$ is an N-series of $\mathrm{IA}_{2 g}$.

6.1. Lower central series of $\mathcal{I}_{g, 1}$. Analogous to Section 5.1 we derive the following results.

Proposition 6.1. $\left\{\operatorname{gr}_{i}\left(\gamma \mathcal{I}_{g, 1}\right)\right\}_{g \in \mathbb{N}}$ gives rise to an $\mathrm{SI}_{\mathbb{Z}}-$ module.

Proposition 6.2. Let $V$ be a rational $\mathrm{SI}_{\mathbb{Q}}$-module which is uniformly representation stable and assume $V_{n}$ is finite dimensional for every $n \in \mathbb{N}$. Then the $k$ th degrees $\mathcal{L}_{k}(V)$ of the free Lie algebra generated by $V$ is a rational $\mathrm{SI}_{\mathbb{Q}}-$ module which is uniformly representation stable and $\mathcal{L}_{k}\left(V_{n}\right)$ is finite dimensional for all $n \in \mathbb{N}$.

Note that the following result can also be derived from the explicit description of Habegger-Sorger [HS00, Thm 1.1].

Theorem 6.3. $\left\{\operatorname{gr}_{i}\left(\gamma \mathcal{I}_{g, 1}\right)\right\}_{g \in \mathbb{N}}$ gives rise to a rational $\mathrm{SI}_{\mathbb{Q}}-$ module.

Proof. Again we have an epimorphism

$$
\mathcal{L}_{i}\left(H_{1}\left(\mathcal{I}_{?, 1} ; \mathbb{Q}\right)\right) \longrightarrow \operatorname{gr}_{i}\left(\gamma \mathcal{I}_{?, 1}\right)
$$

of $\mathrm{SI}_{\mathbb{Z}}$-modules. Because $\mathcal{L}_{i}\left(H_{1}\left(\mathcal{I}_{\text {?,1 }} ; \mathbb{Q}\right)\right)$ is also a rational $\mathrm{SI}_{\mathbb{Q}}$-module, we get a unique rational $\mathrm{Sp}_{2 g} \mathbb{Q}$-representation structure on $\operatorname{gr}_{i}\left(\gamma \mathcal{I}_{g, 1}\right)$ that restricts to the given $\mathrm{Sp}_{2 g} \mathbb{Z}$-representation. Therefore

$$
\mathcal{L}_{i}\left(H_{1}\left(\mathcal{I}_{g, 1} ; \mathbb{Q}\right)\right) \longrightarrow \operatorname{gr}_{i}\left(\gamma \mathcal{I}_{g, 1}\right)
$$

is $\mathrm{Sp}_{2 g} \mathbb{Q}$-equivariant. We can then as in the proof of Theorem 5.3 lift the $\mathrm{SI}_{\mathbb{Z}^{-}}$ module structure to an $\mathrm{SI}_{\mathbb{Q}^{-}}$-module structure.

Proof of Theorem $G$. Let $V$ be the $\mathrm{SI}_{\mathbb{Q}}$-module from Theorem 6.3 such that $V_{g}=$ $\operatorname{gr}_{i}\left(\mathcal{I}_{g, 1}\right)$. Then by its description as an $\mathrm{SI}_{\mathbb{Z}}-$ module it restricts to the $\mathrm{FI}$-module described in [CEF15, Ex 7.3.6]. 
We will use the result [CEF15, Thm 7.3.7] that $V$ is a finitely generated FImodule and thereby a finitely generated $\mathrm{SI}_{\mathbb{Q}}-$ module.

Our Theorem B implies then that $V$ is uniformly representation stable.

6.2. Johnson filtration of $\mathcal{I}_{g, 1}$. Next we consider the Johnson filtration $\alpha \mathcal{I}_{g, 1}$ of the Torelli subgroups $\mathcal{I}_{g, 1}$. The proof of Proposition 5.4 can be used to prove the following analogue.

Proposition 6.4. $\left\{\operatorname{gr}_{i}\left(\alpha \mathrm{IA}_{n}\right)\right\}_{n \in \mathbb{N}}$ gives rise to a $\mathrm{VIC}_{\mathbb{Z}}-$ module.

Similar to the Johnson filtration of $\mathrm{IA}_{n}$, we also get information from the (original) Johnson homomorphism. As explained by Satoh [Sat16, Sec 8] there is an $\mathrm{Sp}_{2 g} \mathbb{Z}$-equivariant monomorphism

$$
\operatorname{gr}_{i}\left(\alpha \mathcal{I}_{g, 1}\right) \hookrightarrow \operatorname{Hom}_{\mathbb{Q}}\left(H_{1}\left(\Sigma_{g, 1} ; \mathbb{Q}\right), \operatorname{gr}_{i+1}\left(\gamma F_{2 g}\right)\right) \cong \mathbb{Q}^{2 g} \otimes \mathcal{L}_{i+1}\left(\mathbb{Q}^{2 g}\right) .
$$

By the same arguments used in the proof of Theorem 6.3, we can deduce the following result.

Theorem 6.5. $\left\{\operatorname{gr}_{i}\left(\alpha \mathcal{I}_{g, 1}\right)\right\}_{g \in \mathbb{N}}$ gives rise to a rational $\mathrm{SI}_{\mathbb{Q}}-$ module.

Proof of Theorem $I$. Let $V$ be the $\mathrm{SI}_{\mathbb{Q}^{-}}$-module from Theorem 6.5 such that $V_{g}=$ $\operatorname{gr}_{k}\left(\alpha \mathcal{I}_{g, 1}\right)$.

Church and Putman [CP15] consider the groups $\mathcal{I}_{g, 1}$ as a weak Fl-group. They apply their [CP15, Thm G] to prove their [CP15, Thm A]. In the proof of the former theorem in [CP15, Claim 2] it is stated that $W(k)$ is boundedly generated. But $W(k)_{g} \otimes_{\mathbb{Z}} \mathbb{Q}$ is $\operatorname{gr}_{k}\left(\alpha \mathcal{I}_{g, 1}\right)=V_{g}$. Because every $V_{g}$ is finite dimensional (see $[\mathrm{CP} 15$, Prop 4.4]), $V$ is a finitely generated $\mathrm{FI}$-module and thus certainly a finitely generated $\mathrm{SI}_{\mathbb{Q}^{-}}$module.

Our Theorem B implies then that $V$ is uniformly representation stable.

\section{REFERENCES}

[And65] S. Andreadakis. On the automorphisms of free groups and free nilpotent groups. Proc. London Math. Soc. (3), 15:239-268, 1965. 4, 29, 34

[Bor60] Armand Borel. Density properties for certain subgroups of semi-simple groups without compact components. Ann. of Math. (2), 72:179-188, 1960. 16, 17

[CEF15] Thomas Church, Jordan S. Ellenberg, and Benson Farb. FI-modules and stability for representations of symmetric groups. Duke Math. J., 164(9):1833-1910, 2015. 1, 2, 19, $20,21,22,26,34,36,37$

[CF13] Thomas Church and Benson Farb. Representation theory and homological stability. Adv. Math., 245:250-314, 2013. 1, 2, 4, 15, 31, 32, 36

[CP15] Thomas Church and Andrew Putman. Generating the Johnson filtration. Geom. Topol., 19(4):2217-2255, 2015. 35, 37

[Dja16] Aurélien Djament. Des propriétés de finitude des foncteurs polynomiaux. Fund. Math., 233(3):197-256, 2016. 34, 35

[FH91] William Fulton and Joe Harris. Representation theory, volume 129 of Graduate Texts in Mathematics. Springer-Verlag, New York, 1991. A first course, Readings in Mathematics. $6,7,8$ 
[Ful97] William Fulton. Young tableaux, volume 35 of London Mathematical Society Student Texts. Cambridge University Press, Cambridge, 1997. With applications to representation theory and geometry. 9

[Gre07] J. A. Green. Polynomial representations of $\mathrm{GL}_{n}$, volume 830 of Lecture Notes in Mathematics. Springer, Berlin, augmented edition, 2007. With an appendix on Schensted correspondence and Littelmann paths by K. Erdmann, Green and M. Schocker. 6

[GW09] Roe Goodman and Nolan R. Wallach. Symmetry, representations, and invariants, volume 255 of Graduate Texts in Mathematics. Springer, Dordrecht, 2009. 6, 9

[GW16] Wee Liang Gan and John Watterlond. Stable decompositions of certain representations of the finite general linear groups. Preprint, 2016, arXiv:1605.08434v2. 4

[Hai97] Richard Hain. Infinitesimal presentations of the Torelli groups. J. Amer. Math. Soc., 10(3):597-651, 1997. 4

[Hön54] Chaim Samuel Hönig. Proof of the well-ordering of cardinal numbers. Proc. Amer. Math. Soc., 5:312, 1954. 27

[HS00] Nathan Habegger and Christoph Sorger. An infinitesimal presentation of the Torelli group of a surface with boundary. Preprint, 2000, http://www.math.sciences.univ-nantes.fr/ habegger/PS/inf180300.ps . 4, 5, 36

[HTW05] Roger Howe, Eng-Chye Tan, and Jeb F. Willenbring. Stable branching rules for classical symmetric pairs. Trans. Amer. Math. Soc., 357(4):1601-1626, 2005. 10

[Jan87] Jens Carsten Jantzen. Representations of algebraic groups, volume 131 of Pure and Applied Mathematics. Academic Press, Inc., Boston, MA, 1987. 6

[Joh80] Dennis Johnson. An abelian quotient of the mapping class group $\mathcal{I}_{g}$. Math. Ann., 249(3):225-242, 1980. 34

[Joh85] Dennis Johnson. The structure of the Torelli group. III. The abelianization of $\mathscr{I}$. Topology, 24(2):127-144, 1985. 35

[Kaw06] Nariya Kawazumi. Cohomological aspects of magnus expansions. Preprint, 2006, arXiv:math/0505497v3. 30

[Koi89] Kazuhiko Koike. On the decomposition of tensor products of the representations of the classical groups: by means of the universal characters. Adv. Math., 74(1):57-86, 1989. $6,11,14,15$

[KT87] Kazuhiko Koike and Itaru Terada. Young-diagrammatic methods for the representation theory of the classical groups of type $B_{n}, C_{n}, D_{n} . J$. Algebra, 107(2):466-511, 1987. $11,12,13$

[Laz54] Michel Lazard. Sur les groupes nilpotents et les anneaux de Lie. Ann. Sci. Ecole Norm. Sup. (3), 71:101-190, 1954. 28, 29

[Nie18] J. Nielsen. Über die Isomorphismen unendlicher Gruppen ohne Relation. Math. Ann., 79(3):269-272, 1918. 29

[PS14] Andrew Putman and Steven V Sam. Representation stability and finite linear groups. Preprint, to appear in Duke Math. J., 2014, arXiv:1408.3694v2. 3, 4, 19

[RWW15] Oscar Randal-Williams and Nathalie Wahl. Homological stability for automorphism groups. Preprint, 2015, arXiv:1409.3541v3. 20

[Sat12] Takao Satoh. On the lower central series of the IA-automorphism group of a free group. J. Pure Appl. Algebra, 216(3):709-717, 2012. 4

[Sat16] Takao Satoh. A survey of the Johnson homomorphisms of the automorphism groups of free groups and related topics. In Handbook of Teichmüller theory. Volume $V$, pages 167-209. Zürich: European Mathematical Society (EMS), 2016. 4, 5, 35, 37

[SSW13] Steven V. Sam, Andrew Snowden, and Jerzy Weyman. Homology of Littlewood complexes. Selecta Math. (N.S.), 19(3):655-698, 2013. 11, 12, 13 
[Wey39] Hermann Weyl. The Classical Groups. Their Invariants and Representations. Princeton University Press, Princeton, N.J., 1939. 6

[Wil14] Jennifer C. H. Wilson. FI $\mathscr{W}$-modules and stability criteria for representations of classical Weyl groups. J. Algebra, 420:269-332, 2014. 1, 2

Institut für Mathematik, Freie Universität Berlin, Germany

E-mail address: peter.patzt@fu-berlin.de 